\title{
Reputación de los medios de comunicación españoles
}

\section{The reputation of the Spanish media}

\author{
Justo Villafañe; Yolanda Ortiz-de-Guinea-Ayala; José-Luis Martín-Sáez
}

Cómo citar este artículo:

Villafañe, Justo; Ortiz-de-Guinea-Ayala, Yolanda; Martín-Sáez, José-Luis (2020). "Reputación de los medios de comunicación españoles". Profesional de la información, v. 29, n. 4, e290407.

https://doi.org/10.3145/epi.2020.jul.07

Artículo recibido el 18-05-2020

Aceptación definitiva: 17-06-2020

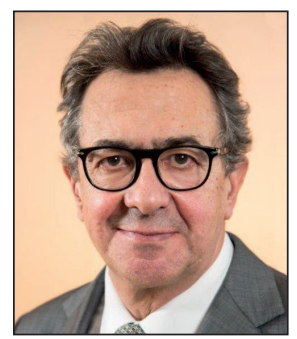

Justo Villafañe $\square$

https://orcid.org/0000-0002-4501-3548

Universidad Complutense de Madrid

Facultad de Ciencias de la Información

Av. Complutense, 3

28040 Madrid, España

justo@villafane.com

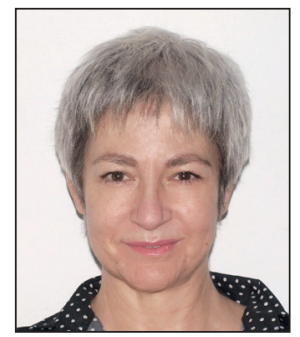

Yolanda Ortiz-de-Guinea-Ayala

https://orcid.org/0000-0001-8560-656X

Universidad Rey Juan Carlos

Facultad de Ciencias de la Comunicación,

Dpto. de Comunicación y Sociología

Camino del Molino, 5

28943 Fuenlabrada (Madrid), España

yolanda.ortizdeguinea.ayala@urjc.es

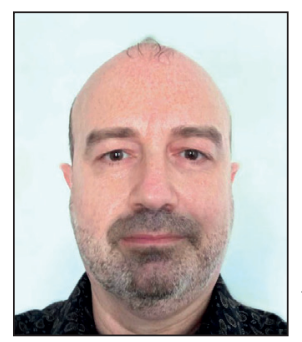

\author{
José-Luis Martín-Sáez \\ http://orcid.org/0000-0002-6979-3470 \\ Universidad Rey Juan Carlos \\ Facultad de Ciencias de la Comunicación \\ Camino del Molino, 5 \\ 28943 Fuenlabrada (Madrid), España \\ joseluis.martin.saez@urjc.es
}

\section{Resumen}

Se han llevado a cabo dos estudios, uno cualitativo y otro cuantitativo, con dos muestras en cada uno. Cada muestra se diferenciaba por el grado de conocimiento del desempeño de los medios de comunicación -población informada y profesionales de la comunicación-. Se realiza un análisis cuantitativo de la reputación de los medios de comunicación de España, a partir de un análisis cualitativo de la realidad profesional y empresarial de los medios españoles con mejor reputación. Se concluye que existe un amplio consenso en que no existe confianza en los medios de comunicación al considerar que la información que difunden está manipulada. La credibilidad de un medio tiene una alta correlación con su trayectoria -algo coherente con la teoría de la reputación-, pues la reputación necesita tiempo para consolidarse como un valor estable. Se valora la reputación de cada tipo de medio - prensa en papel, prensa digital, radio y televisión-. Se incluyen dos rankings con los 16 medios con mejor reputación en España, correspondientes a las dos muestras citadas.

\section{Palabras clave}

Medios de comunicación; Epistemología de la reputación; Reputación mediática; Reputación corporativa; Valor periodístico; Valor empresarial; Variables de reputación mediática; Grupos de interés de un medio; Tipología de medios; Manipulación; Credibilidad; Rigor informativo; Independencia.

\section{Abstract}

Two studies, one qualitative and the other quantitative, have been carried out, each using two samples differentiated by the degree of knowledge of the performance of media: informed population and communication professionals. A quantitative analysis of the reputation of the Spanish media is carried out, based on a qualitative analysis of the professional and business reality of the media with the best reputation. It is concluded that there is a broad consensus that there is 
no confidence in the media because the information that they disseminate is manipulated. The credibility of a medium shows a high correlation with its history, consistent with the theory of reputation, because consolidation of a stable reputation requires time. The reputation of each type of media (paper press, digital press, radio, and television) is evaluated, yielding two rankings of the 16 media with the best reputation in Spain, corresponding to the two samples analyzed.

\section{Keywords}

Media; Reputation epistemology; Media reputation; Corporate reputation; Journalistic value; Business value; Media reputation variables; Media stakeholders; Media typology; manipulation; Media types; Credibility; Informative rigor and independence.

\section{Introducción}

Pocos sectores empresariales con una influencia tan directa e importante sobre la sociedad han sufrido un proceso de transformación y depreciación -axiológica y económica- como el de los medios de comunicación, especialmente en Europa en las últimas dos décadas. Sus causas son diversas y sus efectos también, pero si intentamos una síntesis de cuál ha sido el resultado de este proceso podríamos afirmar que la pérdida de confianza de la sociedad en los medios de comunicación, y en la función que históricamente se les atribuyó, es la más determinante de todas ellas.

Las nociones de confianza y reputación, como se verá a lo largo de este texto, están íntimamente asociadas. Esta es la razón por la que se ha elegido la Teoría de la Reputación Corporativa, como paradigma epistemológico para ahondar en la crisis de los medios en España y en diez países europeos. Previamente al inicio del estudio, a principios de 2018, ya se había testado el método por un miembro del actual equipo de investigadores (Ortiz-de-Guinea-Ayala, 2015), cuyos resultados confirmaron tanto la pertinencia del objeto de investigación como una buena parte de la metodología que ulteriormente seguimos en la actual investigación, la cual superó la selección del Plan Nacional de Investigación de 2017 y cuyo desarrollo se inició en 2018 y culminará al final de 2020.

En una aproximación iniciática a este proceso crítico que ha cambiado la faz de los medios de comunicación en todo el mundo, pero muy especialmente en Europa, observamos unos factores intrínsecos y otros exógenos que pueden contribuir a explicar sus consecuencias.

Entre los primeros, el cambio tecnológico supuso una transformación radical en los modos de hacer de los medios; aunque no más que en otras industrias o sectores que también han caminado por esa transformación digital y con efectos, por todos conocidos: nuevos modelos de negocio, de producción -con los consiguientes ajustes de plantilla en las empresas editoras y audiovisuales-, cambios en el consumo de los contenidos generales, pero también en los informativos. En resumen, los medios mutaron las formas de intermediación social.

Como consecuencia de estas y otras transformaciones, se creó un nuevo ecosistema mediático en Europa en el que las empresas editoras o audiovisuales que continuaron liderando la pirámide mediática han desarrollado nuevas formas de distribución y de acercamiento a sus audiencias tratando en lo posible de ofrecerles contenidos más personalizados, mientras que dichas audiencias por el contrario filtran y seleccionan cada vez más, y sin que esté muy claro en función de qué criterios, la sobre oferta de información, añadiendo a la de los medios y, obviamente, en detrimento de estos, aquella otra que les llega a través de las redes sociales.

Entre las múltiples causas exógenas que han afectado a los medios, algunas de las cuales por su concreción serán revisadas con más detalle al final del siguiente epígrafe, destacamos otras dos crisis como causa de la crisis mediática: la económica y la de las democracias liberales en numerosos países. La primera, datada en 2007 en Estados Unidos y un año más tarde en Europa, supuso el ajuste ya mencionado en el sector de los medios de comunicación, especialmente en Europa.

La crisis de la democracia, no por diferente en cuanto a su naturaleza ha tenido menor impacto en el ecosistema mediático. La confianza de la población en los medios de comunicación sigue siendo la más baja entre las instituciones que anualmente evalúa el Trust barometer en su edición de 2020 (figura 1).

Solo un $47 \%$ de la población general confía en los medios de comunicación, idéntico porcentaje que en los gobiernos y un $8 \%$ por debajo de las empresas y las ONGs. Preguntado el público informante por la calidad de la información de los medios que habitualmente consumen, el 57\% afirmó que eran indignos de su confianza y un $76 \%$ se mostró preocupado por las noticias falsas que utilizaban como armas. Este porcentaje ha crecido un $2 \%$ en el pe-

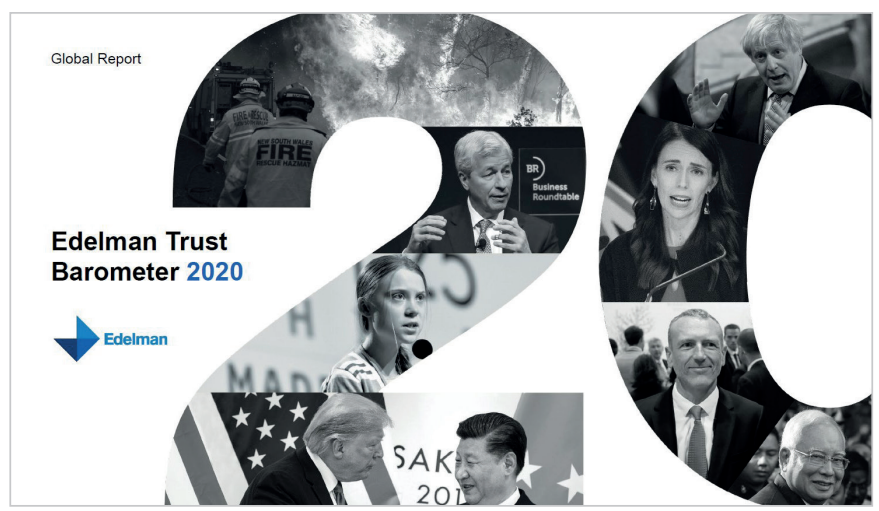

Figura 1. Trust barometer. https://edl.mn/2NOw/tm 
El marco teórico de la presente investigación contiene tres partes diferenciadas, que se desarrollan sucesivamente. En primer lugar se formulará el estatuto epistemológico de la Teoría de la Reputación Corporativa, que se constituye en el marco teorético de dicha investigación. En segundo lugar nos ocupamos del estado del arte dentro de la teoría de la reputación, no solo aplicada a la empresa sino a otros objetos de estudio desde esta perspectiva reputacional, y en la que principalmente buscamos contrastar nuestras herramientas conceptuales y teóricas sobre la reputación con las de otros académicos. Finalmente, este análisis del estado del arte se focaliza, específicamente, en las aportaciones científicas, escasas pero existentes, referidas a la reputación de los medios de comunicación.

Como se ha dicho, la investigación se inscribe dentro del marco teórico de la reputación corporativa, una disciplina que dentro de las ciencias sociales ha logrado un estatuto epistemológico suficientemente robusto -como a continuación se tratará de demostrar- que nos evita tener que acudir a planteamientos multidisciplinares no del todo precisos dado el objeto científico tan concreto del presente estudio: la reputación de los medios de comunicación. Sí es cierto que acudimos con alguna frecuencia a disciplinas como la Teoría de la Comunicación, en la cual nos hemos inspirado en la tercera parte de este marco teórico al abordar el estado del arte sobre el análisis reputacional de los medios de comunicación y su evolución en las últimas dos décadas.

La Teoría de la Reputación Corporativa ha adquirido una madurez epistemológica en el sentido que Bunge (1985) exige a una teoría científica de basarse en una investigación de sus fundamentos y justificarlos en función de sus componentes epistemológicos.

La teoría de la reputación es una teoría factual, se basa en hechos y, en consecuencia, su análisis debe convalidarse empíricamente para poder explicar esos hechos. Como toda teoría factual, la de reputación se asienta en una base conceptual y epistemológica a partir de la cual se pueden establecer sus fundamentos: los presupuestos de los que parte, las nociones primitivas que la teoría adopta, aquellos conocimientos que convierte en axiomas y las reglas de interpretación que utiliza. Ortiz-de-Guinea-Ayala, Villafañe y Caffarel (2018) ya avanzan dicho estatuto al que nos adherimos en la mayoría de sus términos.

Los presupuestos de los que parte la teoría reputacional son:

- La reputación es el reconocimiento que hacen los grupos de interés de una organización de su realidad y de su comportamiento en función del grado de satisfacción de sus expectativas. Por organización la teoría de la reputación puede referirse -como es el caso más frecuente- a una empresa, pero también a cualquier otra entidad.

- El reconocimiento de la realidad y el comportamiento de la organización exige grupos de interés informados que construyan juicios y valoraciones solventes, más allá de las simples percepciones.

- La reputación de la organización debe ser verificable empíricamente ya que se basa, en primer lugar, en la realidad y el comportamiento.

Las nociones primitivas en las teorías factuales deben cumplir algunas reglas de validez:

- la primera es la necesaria extensión de conocimiento que permita su asociación con significados y nociones de otras disciplinas constituyendo un conocimiento nuevo propio de la teoría factual que se está formalizando epistemológicamente;

- en segundo lugar, requieren hondura suficiente para encontrar y establecer relaciones con otras nociones del objeto de análisis que enriquezcan la teoría factual;

- por último, deben poseer un carácter genérico y omnicomprensivo capaz de analizar casos diferentes pero que debido a la hondura de la noción que se acaba de citar, permitan conexiones de significado en un nivel más profundo.

Las nociones primitivas de la teoría de la reputación más pertinentes se resumen a continuación:

- La noción canónica de la reputación que apunta la existencia de un paradigma de reputación ligado a su objeto y que establece unos componentes o dimensiones básicas del objeto de la reputación. En el caso de la noción de reputación corporativa algunas de estas dimensiones básicas o valores de reputación de una empresa son la calidad de su oferta comercial, los resultados económico financieros, el talento de su plantilla, la ética y la sostenibilidad, la innovación...

- La noción de jerarquía, que establece un orden de prelación entre todos los componentes de la reputación: valores, variables u objetivos de reputación, indicadores mensurables empíricamente..., gracias a los cuales será posible la evaluación empírica del objeto de la reputación.

- La noción de variabilidad de los componentes de la reputación que afirma la distinta importancia de los valores reputacionales de ellos derivados y de los grupos de interés afectados, propios del objeto de la reputación. Al combinar estas dos escalas jerárquicas-importancia de los valores de reputación y de los grupos de interés- la conclusión respecto a un medio de comunicación, por ejemplo, será el resultado, al menos, del producto de la importancia que tenga un valor de la reputación mediática por la importancia de un público para ese medio. La noción de variabilidad nos parece más pertinente que la de estratificación que es una de las nociones primitivas de la teoría de la reputación apuntadas por Ortiz-de-Guinea-Ayala, Villafañe y Caffarel (2018). 
- Finalmente, entendemos que la teoría de la reputación posee una primitiva que es su dimensión axiológica, en la medida en que la buena reputación de cualquier sujeto implica lo que en dicha teoría se denomina el binomio reputacional, cuyo primer elemento será un buen comportamiento, meritorio y sostenido en el tiempo, por parte del sujeto, un cumplimiento por encima de aquello a lo que le obliga la ley, y cuyo segundo elemento lo constituye el reconocimiento de ese comportamiento por parte de sus grupos de interés clave cuando satisface las expectativas de estos.

La teoría de la reputación, como toda teoría factual, se apoya en algunos axiomas -conocimientos, no necesariamente demostrados previamente, sobre los cuales la teoría se fundamenta para formular sus propios conceptos-aunque no es bueno axiomatizar demasiado conocimiento porque la fiabilidad de la teoría se resiente.

- El primer axioma es que la reputación está, siempre, ligada a la realidad, lo que descarta la identificación o asimilación del concepto de reputación con cualesquiera otros como los de imagen, percepción, responsabilidad...

- El segundo axioma, derivado del primero, es que la reputación es verificable empíricamente por técnicas que puedan objetivar, generalmente de manera estadística, el componente de realidad y, a través de técnicas convencionales, como los estudios cualitativos o las encuestas, el segundo componente-el reconocimiento de sus grupos de interés- y que el resultado de esa evaluación empírica tendrá en cuenta las diferentes escalas de importancia resultantes de la noción primitiva de jerarquía.

- El tercer axioma de la teoría de la reputación exige que en el análisis del reconocimiento se cuente con población informada y, dependiendo de la complejidad del objeto al que se aplique la teoría de la reputación, con población experta.

El componente último de la investigación de fundamentos de una teoría son sus reglas de interpretación, las cuales dan un sentido y un significado al resto de los componentes - principalmente a sus nociones primitivas- y a todos los conceptos que el desarrollo vaya formalizando hasta alcanzar su estatuto epistemológico propio como, entendemos, ya ha hecho la Teoría de la Reputación Corporativa que constituye el marco teórico en el que se inscribe esta investigación.

Obviamente, en esta segunda parte del marco teórico, nos vamos a centrar en la comunidad científica internacional y en la cada vez más prolífica producción de estudios e investigaciones, muchos de los cuales -como esta aportación-con resultados demostrados con evidencias empíricas una vez resueltos los problemas epistemológicos a los que hasta ahora no se había dedicado demasiada atención. Veamos, a continuación, una necesaria síntesis de estas aportaciones académicas, en la cual tampoco están ausentes las contribuciones profesionales, ya que una buena parte del conocimiento empírico sobre la reputación proviene de grandes consultoras internacionales que tratan de objetivar ante sus clientes el retorno económico de la reputación de las empresas.

El estado del arte de la teoría de la reputación, como ya se ha advertido, está muy orientado hacia el ámbito empresarial, aunque cada vez son más diversos los ámbitos de aplicación y las aportaciones tanto desde el mundo académico como desde el profesional.

Una de estas aportaciones pioneras en el estudio de la reputación corporativa es la de Fombrun (1996) quien, aunque no diferencia las nociones de reputación y percepción o imagen, sí atribuye a aquellas organizaciones que gozan de una buena reputación ventajas que se reflejan, por ejemplo, en el atractivo de sus productos para los consumidores, por un lado, y en su atracción como empleadores y clientes deseados para trabajadores y proveedores, respectivamente, por otro. La reputación corporativa, además, expande sus efectos favorables a largo plazo, como señalan Roberts y Dowling (2002) o Choi y Wang (2009), al facilitar altos rendimientos sostenidos en el tiempo, así como la recuperación después de periodos de crisis, un fenómeno que Weick et al. (2008) llaman resiliencia organizativa. La buena reputación incrementa, por tanto, el potencial de una empresa a la hora de mantener unas relaciones favorables con sus respectivos grupos de interés y, en consecuencia, la gestión de la reputación corporativa se ha convertido en una parte esencial dentro del organigrama de las organizaciones, como señalan Cravens et al. (2003).

Un hecho que ha provocado que en los últimos años el número de publicaciones sobre el estudio de la reputación corporativa en revistas con un gran índice de impacto haya aumentado de forma considerable. Este aumento reflejaría, por otra parte, un estadio protocientífico en el estudio de esta materia, como señalan algunos autores (Walker, 2010; Lange et al., 2011), que se correspondería a su vez con que muchas de estas publicaciones son estudios de revisión bibliográfica, que tienen como objetivo fundamental clasificar y organizar todo lo publicado sobre este asunto, tal y como afirman, entre otros, Roberts y Dowling, 2002; Villafañe, 2004 y 2013; Berens y Van Riel, 2004; Lange et al., 2011; Raithel y Schwaiger, 2015. Publicaciones que ponen de manifiesto, igualmente, la falta de acuerdo sobre la definición del concepto de reputación corporativa. Un concepto formulado por Fombrun (1996) como

"la representación de las percepciones sobre las acciones pasadas de una empresa y sus perspectivas de futuro que describen de forma global el atractivo de esta para sus principales grupos de interés en comparación con otros rivales destacados"

y que, como tal, se convierte en el punto de inicio de un debate en el que se apuesta por un acercamiento a dicho concepto desde la perspectiva de la percepción. Lo que significa que la reputación de las organizaciones reside y se define en las percepciones que los grupos de
Aunque el cambio tecnológico se cita habitualmente como el origen de la crisis del sector, la responsable es la pérdida de valores periodísticos 
interés tienen sobre ellas. Este carácter perceptual sobre la reputación se acepta en otros estudios posteriores (Wartick, 2002), donde además se hace una reflexión crítica sobre la definición y medida de la reputación.

Ahondando en este debate, pero ya desde una perspectiva experiencial, Villafañe determina que la reputación es

"la cristalización de la imagen corporativa de una entidad cuando ésta es el resultado de un comportamiento corporativo excelente, mantenido a lo largo del tiempo, que le confiere un carácter estructural ante sus stakeholders estratégicos" (Villafañe, 2004).

De este modo, son los grupos de interés quienes determinan la reputación de una organización a partir de distintas variables reputacionales, de acuerdo con la experiencia acumulada que tienen en su relación con dicha organización. Se puede hablar así, por un lado, de reputación, como idea que se construye a lo largo del tiempo a partir de la actuación de una empresa, que vincularíamos con esta perspectiva experiencial y, por otro, de imagen, que no es más que una valoración que se tiene a partir de una determinada percepción, no necesariamente informada, vinculada con la visión perceptual.

Dos trabajos más recientes analizan las aportaciones hechas en los últimos años acerca del concepto de reputación corporativa. En el primero de estos trabajos Walker (2010) hace una revisión bibliográfica con el objetivo de determinar qué es y qué no es la reputación corporativa, partiendo del estudio de Wartick (2002), además de diferenciar perfectamente los conceptos de identidad, imagen y reputación de una empresa. Walker (2010) advierte, por otro lado, de la falta de profundidad de la construcción teórica desarrollada acerca de este asunto hasta ese momento.

En el segundo de estos trabajos Lange et al. (2011), partiendo de las definiciones de reputación corporativa hechas con anterioridad, identifican tres dimensiones sobre este concepto:

- el ser conocido, idea que hace referencia a la notoriedad de la empresa, sin que esto suponga una opinión sobre la reputación de la misma;

- el ser conocido por algo, noción que tiene que ver con la capacidad de prever cómo va a actuar la empresa en relación con un determinado público y sus intereses, lo que llamaríamos reconocimiento; y

- la percepción general favorable, materializada en las visiones de un conjunto concreto de agentes sobre una determinada empresa, y que denominaríamos atractivo.

Esto quiere decir que la reputación corporativa se caracteriza por el grado de conocimiento con que cuenta una determinada organización y, por ende, la experiencia y la relación que se han tenido con ella; las creencias sobre lo que se espera en el futuro de dicha organización y las percepciones sobre el atractivo general de la misma.

Estos mismos autores, dado que la mayoría de los trabajos que analizan la definición de reputación corporativa, así como las formas de su medición, no parten de una teoría concreta, se plantean además cuáles son los fundamentos epistemológicos del estudio de dicho concepto. Así, tanto Lange et al. (2011) como, por ejemplo, McWilliams y Siegel (2001), Roberts y Dowling (2002), Martín-de-Castro et al. (2006) y McWilliams et al. (2006) se apoyan en la llamada teoría de recursos y capacidades, al considerar que la reputación corporativa es, probablemente, el recurso más importante con el que cuenta una empresa. Un recurso que, como elemento intangible, comparte con la mayoría de los elementos de su misma naturaleza el largo y lento proceso de acumulación, así como la dificultad de imitación. La reputación, en este sentido, como se encargan de señalar Castelo y Lima (2006), se nos presenta como un recurso de extraordinaria utilidad para mejorar la relación de una empresa con sus correspondientes grupos de interés: clientes, inversores, proveedores y competidores.

De-la-Fuente-Sabaté y De-Quevedo-Puente (2003) proporcionan un segundo basamento epistemológico a la hora de abordar el estudio de la reputación corporativa, en este caso, partiendo de la idea de la consideración de la empresa como nexo de contratos. Originalmente presentada por Jensen y Meckling (1976) y desarrollada más tarde por Cornell y Shapiro (1987) y Hill y Jones (1992), esta idea considera la reputación corporativa como una garantía contractual, que se define por lo que se espera de una empresa por parte de los grupos de interés con los que se relaciona. De este modo, la reputación de una empresa se verá dañada, si su forma de actuar es contraria a lo que un determinado agente espera de la misma cuando interactúa con ella.

Montero et al. (2011) establecen, finalmente, un paralelismo entre el concepto de reputación corporativa y el de capital social, a partir de la definición que hacen de este último concepto Nahapiet y Ghoshal (1998), para quienes capital social es

"la suma de los recursos actuales incluidos, disponibles o derivados de la red de relaciones que posee un individuo o una unidad social".

La reputación corporativa se identifica, en consecuencia, con las relaciones favorables que una empresa establece con otras organizaciones o individuos y que le conducen potencialmente a la creación de un mayor valor (Arregle et al., 2007). Para estos autores, por tanto, las ventajas que tiene una empresa con una buena reputación corporativa son prácticamente las mismas, si no todas, que las que tendría si tuviera un gran capital social.

En cualquier caso, todos los que trabajan en el campo de la reputación corporativa están de acuerdo en la importancia de esta en la empresa y, por tanto, coinciden también en la necesidad de medirla de la forma más precisa posible, ya que esta es la mejor manera de gestionarla. Porque lo que no se mide con precisión, no se puede gestionar con eficacia. 
Sin embargo, a la hora de medir la reputación corporativa nos enfrentamos a algunos de los obstáculos mencionados anteriormente. El principal de estos obstáculos es, precisamente, la falta de consenso a la hora de definir este concepto (Mahon, 2002; Wartick, 2002; Walker, 2010). Una falta de acuerdo en la definición que tiene como consecuencia, lógicamente, la falta de acuerdo a su vez en una forma de medición universal.

Este desacuerdo en una forma de medición universalmente aceptada se refleja en toda una serie de diferentes métodos de medir que, sin embargo, son menos numerosos que el glosario de definiciones. Este hecho se convierte también en un obstáculo a la hora de estudiar la reputación corporativa. Las dificultades técnicas y los costes propios de los procesos de medición han llevado a que los estudiosos del tema se limiten, con frecuencia, a exponer cómo debería hacerse la medición o a valorar simplemente uno o varios aspectos de dichos procesos de medición, obviando estudiar y analizar el asunto globalmente. Porque con independencia de que nos aproximemos al estudio de la reputación corporativa desde una visión perceptual o experiencial, el hecho de que esté fundada en la relación de una empresa con sus grupos de interés, su evaluación conlleva recabar toda la información posible de estos stakeholders en numerosas variables -éticas, laborales, financieras o de otro tipo-, así como su comparación con estándares propios o externos. Unas acciones que conllevan, lógicamente, unos grandes costes.

En consecuencia, frecuentemente, las llamadas herramientas de "medición de la reputación" se circunscriben a verificar el cumplimiento de una serie de estándares en determinados ámbitos; a la tenencia de reconocimientos o sellos de calidad; a la percepción que tienen los clientes o usuarios de las empresas y servicios, o a la responsabilidad social que tienen los mismos.

Así Berens y Van Riel (2004) hablan de tres grandes modos de medir la reputación corporativa:

- en primer lugar, de los rankings, asociados a las expectativas que tienen los distintos agentes sobre cómo deben comportarse las empresas;

- en segundo lugar, de la confianza, vinculada a la percepción que se tiene de la benevolencia, fiabilidad y honestidad de una organización (Newell; Goldsmith, 2001) y,

- en tercer lugar, de las medidas de personalidad corporativa, que parten de los estudios de Davies et al. (2015).

También distinguen tres tipos de mediciones Nájera et al. (2014):

Por un lado, señalan la existencia de un determinado número de estudios que, partiendo de la crítica a trabajos y rankings anteriores, proponen una metodología original, cuyas herramientas diseñadas se utilizan, generalmente, una sola vez y de forma específica en una empresa (Cravens et al., 2003; De-la-Fuente-Sabaté; De-Quevedo-Puente, 2003; Helm, 2007). Sin embargo, y a pesar su solidez científica, estas herramientas se utilizan muy pocas veces, debido a sus altos costes y los problemas técnicos que plantea su implementación.

- Por otro lado, los citados autores hablan de las medidas parciales que se centran en la medición y valoración de aspectos concretos de una organización -generalmente, la marca-, que se basan en escalas ya utilizadas (Caruana; Chircop, 2000) y/o en rankings como el de Interbrand.

https://www.interbrand.com/best-brands/best-global-brands/2018/ranking

Medidas que son cuestionadas, por otra parte, por autores como Chun (2005), quien, partiendo de la base de que los conceptos de marca y reputación no son análogos, advierte que estas herramientas no sirven en la práctica para medir la reputación corporativa, ya que habitualmente utilizan información de un único grupo de interés, sin tener en cuenta el carácter global intrínseco de la reputación corporativa, al que hacen referencia la inmensa mayoría de sus definiciones.

Nájera et al. (2014) hablan, por último, de los rankings o monitores. Unas herramientas que son multidimensionales, pero que no siempre son multi-stakeholders, y que presentan, como gran debilidad, la falta de una base epistemológica explícita, tanto en su desarrollo como en su origen (De la Fuente-Sabaté; De Quevedo-Puente, 2003). Hasta el punto de que en muchos casos, como ocurre con el conocido Fortune WMAC, por ejemplo, no parten de una definición concreta de la construcción teórica que tratan de medir. A pesar de ello, tal y como señala Walker (2010), estos sistemas de medición constituyen la fuente con mayor credibilidad a la hora de conocer cuál es la reputación corporativa de una empresa. En este sentido, cabe destacar aquí las herramientas de medición desarrolladas por Reputation Institute o el Monitor Empresarial de Reputación Corporativa (Merco), diseñado por Villafañe (2000) y publicado en España desde el año 2001, y que actualmente está presente en otros once países.

El hecho de que existan varias herramientas dentro de la categoría de monitores y rankings puede generar dudas acerca de cuáles son los aspectos de los mismos que resultan de mayor utilidad para las empresas. Villafañe (2013), en este sentido, evalúa estos instrumentos a partir de un modelo basado en cuatro criterios:

- en primer lugar, el rigor metodológico, tratando de conseguir un sistema de medición que se ajuste lo más perfectamente posible al objeto que se pretende medir;

- en segundo lugar, la influencia económica y empresarial, que se refleja en la repercusión que los resultados de ese ranking o monitor pueden tener en los grupos de interés de una empresa, en el número de países en los que se elabora ese monitor y en el grado de interacción resultante en la empresa durante el proceso de elaboración de dicho ranking;

- en tercer lugar, la transparencia, que se corresponde con un proceso de evaluación claro, la verificación externa del mismo y la publicación de sus resultados; y 
- en cuarto lugar, la repercusión mediática que tienen los resultados publicados de ese ranking o monitor.

Criterios que, a nuestro juicio, deben ser los fundamentos en los que debe apoyarse cualquier nuevo instrumento de medición de la reputación corporativa, como la que, en el caso que nos ocupa, este proyecto pretende diseñar acerca de los medios de comunicación.

Para establecer los límites de la investigación y concluir con el marco teórico en el que ésta se inscribe vamos a revisar, finalmente, las escasas pero importantes aportaciones empíricas que sobre la reputación de los medios de comunicación han aparecido en los últimos años y a los que este texto pretende contribuir.

Ya se han comentado recientes datos sobre la situación crítica y la pérdida de confianza y, consiguientemente, de reputación que los medios de comunicación han sufrido en las últimas dos décadas; todo ello agravado por los efectos de la crisis financiera, económica y social que en forma de precariedad salarial, calidad del empleo, recorte de plantillas y cierre de medios, ha contribuido a que el sector de medios de comunicación sea, probablemente, el sector económico más castigado desde la pasada crisis. Entre 2008 y 2015 los medios de comunicación en España destruyeron 12.200 puestos de trabajo (Informe Anual de la Profesión Periodística, 2015).

Aunque el cambio tecnológico se cita habitualmente como el origen de la crisis del sector, no es exactamente la transformación digital la responsable de dicho cambio sino la pérdida de valores periodísticos que a veces se ha querido justificar con la digitalización no siendo ésta la única -ni la más importante- de las razones que lo produjeron, sino la desregulación del ecosistema mediático producido por internet. Este hecho ha sido suficientemente documentado (Madden; Smith, 2010; Aula, 2010; Del-Fresno-García, 2011; Fondevila-Gascón et al., 2012; Ramírez-de-la-Piscina et al., 2014) lo que nos exime ahora de abundar en el mismo.

La intrusión de internet y de las redes sociales cambió radicalmente el uso y consumo de la información; la aparición de los nuevos nativos digitales y las redes como canales de información alternativos, coincidiendo con el cambio de modelo de negocio de los medios tradicionales y la crisis económica de 2008, constituyeron un caldo de cultivo decisivo para la pérdida de reputación de los medios tradicionales, aunque el propio concepto aún no estuviera acuñado y se identificara con el de credibilidad (Metzger et al., 2003), quizá el desiderátum de lo se ha considerado hasta ahora un medio de calidad, o un medio de referencia, y sobre el que más instrumentos e índices de evaluación han centrado su atención (Trumbo; McComas, 2003).

Llegado este punto -y para distinguir credibilidad de reputación- es necesario avanzar un concepto de reputación mediática, de acuerdo con el paradigma de la Teoría de la Reputación Corporativa. En este sentido la reputación de un medio de comunicación es el reconocimiento que los grupos de interés de dicho medio hacen de su comportamiento profesional y empresarial, en función del grado de satisfacción de sus expectativas (Ortiz-de-Guinea-Ayala, 2015). Esta definición incluye, no por vez primera (Deephouse, 2000), pero sí de manera inhabitual la dimensión empresarial como una parte consustancial al buen desempeño, a la reputación, en suma, de un medio de comunicación que va más allá del valor periodístico y requiere para ser cabalmente entendido -al menos desde la perspectiva de la teoría de la reputación- de un valor empresarial que garantice su sostenibilidad económica (Murphy, 2010).

Otro de los cambios experimentados en el ecosistema de los medios en España durante la última década y media es el achatamiento de la pirámide mediática, en la que aparecen muy pocos medios de referencia en el vértice superior y un ensanchamiento, sin precedentes, en la nueva base mediática (Canavilhas, 2011), donde coexisten empresas informativas en crisis, nuevos nativos digitales, medios locales, especializados... Un ecosistema donde los emisores de información tratan de encontrar canales eficaces, y eficientes, de conexión con las audiencias y éstas, como si la teoría de Mauro Wolf sobre el gatekeeping (Villafañe; Bustamante; Prado, 1987) hubiera revivido, tratan de seleccionar la excesiva demanda informativa que tienen a su disposición.

Los medios de comunicación, inmersos en una crisis sin precedentes en su historia, necesitan ahora rearmarse con los mismos valores que en su día legitimaron su función social. Este componente axiológico junto con la imprescindible sostenibilidad económica -ligada a su independencia, un valor periodístico y a la vez empresarial- es la base de la confianza, la única posición desde la que se puede obtener una licencia social para operar que, en el caso de los medios de comunicación, no es otra que la formación de una opinión pública cívica y cosmopolita (Luhmann, 2007) desde la cual renovar sus categorías de intermediación social.

\section{Metodología}

La metodología empleada en la investigación ha combinado diversas técnicas cualitativas y cuantitativas que se aplicaron en primer lugar en España -durante el segundo semestre de 2018 y el primero de 2019-y posteriormente, cuando los resultados obtenidos se consideraron satisfactorios, se procedió a replicar la misma metodología -aunque con una menor dimensión que en el caso español- en los diez países que componían la muestra europea. Dicha metodología 
comprendió las siguientes cinco etapas ya que el estado del arte, que generalmente constituye la primera se ha incluido -como acabamos de ver- en el marco teórico y la séptima y última -las conclusiones de las diversas técnicas de investigación empleadas- se desarrolla, detalladamente, en el siguiente epígrafe de este texto dedicado a los resultados y conclusiones de la investigación.

- Formulación de las preguntas de investigación.

- Estudio cualitativo sobre la reputación de los medios de comunicación en España.

- Definición del universo y selección de la muestra de análisis en España.

- Análisis cuantitativo de la reputación de los medios de comunicación.

- Análisis de la realidad profesional y empresarial de los medios de comunicación con mejor reputación en España.

La primera etapa del estudio fue la elaboración de las preguntas de investigación que, tras la información obtenida en el estudio del arte y de la revisión teórica efectuada, formulamos de manera explícita con los siguientes enunciados:

- ¿Cuáles son los valores que determinan la reputación de un medio de comunicación?

- ¿Qué importancia posee cada uno de los valores de la reputación mediática?

- ¿Cuáles son los grupos de interés más importantes de un medio de comunicación en España atendiendo a los valores de reputación mediática identificados?

- De acuerdo con la aplicación de la Teoría de la Reputación Corporativa, no solo a las empresas, sino también a otros objetos de análisis como las ciudades, instituciones... ¿se puede elaborar un 'árbol de variables' de reputación mediática con un significado más reducido, y mono sémico, dependientes de los valores de reputación mediática obtenidos previamente?

- ¿Qué indicadores se podrían asociar a cada variable de reputación mediática identificados en la pregunta anterior?

- ¿Qué valoración de la reputación mediática obtienen los cuatro tipos de medios -prensa escrita, prensa nativa digital, radio o televisión-analizados en esta investigación?

La indagación inicial no podía hacerse sino a través de un estudio cualitativo que para garantizar la no contaminación del discurso por la posible influencia de opiniones expertas, se inició a partir de dos grupos de discusión a población informada y, seguidamente, se hicieron 22 entrevistas en profundidad, ahora sí, a diversos tipos de profesionales de los medios de comunicación, entre los que un tercio eran directores de un medio y entre los que había también editores, gerentes y periodistas con experiencia en medios de referencia, además, de varios directores de comunicación de grandes compañías españolas.

Los dos focus groups con población informada se segmentaron en función de la edad de los participantes cubriendo dos segmentos de edad -25 a 39 y 40 a 55 años- para evaluar la opinión de la población informada respecto a los medios de comunicación en España y aproximarnos gradualmente a lo que entendían por reputación mediática.

A partir de los primeros resultados del estudio cualitativo, se procedió a la definición del universo y selección de la muestra de análisis cuantitativo en nuestro país mediante esas 22 entrevistas en profundidad a profesionales, para validar el objeto, los límites de la investigación y tratar de identificar los valores y variables de la reputación mediática, así como su propio juicio sobre cuáles eran los medios con mejor reputación en España.

No obstante, y para dotar de mayor consistencia a las conclusiones obtenidas tras las entrevistas efectuadas, y especialmente la muestra de medios seleccionada, posteriormente se efectuó una consulta por cuestionario a 135 profesionales de los medios de comunicación -periodistas y gestores-y a nuevos dircoms, pidiéndoles que incluyeran, si lo consideraban necesario, a otros medios que no aparecieran en la primera selección efectuada y que, a su juicio, gozaran de una reputación destacable.

El ulterior análisis cuantitativo de la reputación de los medios de comunicación de la muestra final se efectuó a través de dos muestras: 135 entrevistas semiestructuradas a periodistas (de medios y dircoms) y a gestores de medios para evaluar su reputación en España, estableciendo un diagnóstico de éstos a partir de los valores y variables de reputación mediática obtenidos en el estudio cualitativo efectuado mediante las 22 entrevistas en profundidad, tanto a nivel general como en los diferentes medios de la muestra. Un estudio final, a través de un cuestionario telefónico, a población informada de 1.000 casos, para analizar el tipo de medio que gozaba de mayor reconocimiento, los valores y variables que determinaban la reputación mediática y la valoración otorgada a cada medio del repertorio final, y definitivo, obtenido en la etapa anterior.

Las primeras cinco etapas de la metodología, enunciadas y descritas anteriormente, sirvieron para obtener una información robusta y rigurosa -cualitativa y cuantitativamente- del reconocimiento que los distintos públicos informantes dispensaron a un grupo de medios de comunicación. El conjunto de tareas que se describen a continuación ocupó desde el segundo semestre de 2018 hasta los primeros meses de 2020 ya que la muestra europea comenzó a evaluarse una vez que fueron confirmados los datos descritos hasta ahora en el ámbito español.

\section{Resultados}

Una vez formuladas las preguntas de investigación se realizó un estudio cualitativo sobre la reputación de los medios de comunicación en España para dotar de contenido y de la suficiente masa crítica a la investigación con el fin de dar respuesta a las citadas preguntas. Para ello, en primer lugar vamos a extractar las claves del discurso social de la población 
informada, obtenido a partir de los dos focus groups realizados. Dado que el de reputación mediática no es un concepto asumido ni conocido, se procedió con mucha cautela preguntando a nuestros informantes por cuestiones iniciáticas muy generales como la información que recibían, los medios que seguían habitualmente y, a partir de ahí, en etapas sucesivas, ir ahondando en las diferentes lógicas argumentales del público informante hasta llegar a clausurar el discurso en torno a la reputación mediática y sus componentes.

Para todos ellos la información transmitida por los medios se percibe como manipulada y partidista. Algo que entre los más jóvenes aparece de manera manifiesta en su discurso, donde la manipulación se asocia principalmente con la gente que manda y decide sobre qué quiere que se entere la ciudadanía. Ello supone que, en general, no se cuenta la verdad y por tanto la información no resulte real. Aun así nuestros/as informantes jóvenes valoran que la información les resulta útil, además de entretenida, aun cuando para corregir de alguna manera su sesgo y su manipulación haya que tener en cuenta de dónde procede la información y cuáles son sus condicionantes personales.

En cuanto a la actitud mantenida en la búsqueda de la información nuestros/as informantes más jóvenes insisten en recalcar ciertas diferencias con respecto a las generaciones anteriores. Se consideran: menos estables en cuanto a las fuentes a consultar y -afirman- consultan fuentes distintas según la información que se busque; es decir, son menos fieles en cuanto a las fuentes a las que acuden; tampoco mantienen fidelidad a unas determinadas fuentes sino que cambian con facilidad de unas a otras. Como consecuencia de esto consumen menos los medios tradicionales, periódicos y televisión, y se procura más la búsqueda de información por otros medios; se busca un cierto protagonismo de las noticias opinando, comentando, rebatiendo... y se diferencia entre los sitios para informarse, los medios, y los espacios para opinar, los foros.

Los informantes más adultos se reiteran en el hecho de sentirse manipulados. Una manipulación que se asume como algo tradicional en nuestro país y que, también en este caso, se atribuye a las élites del poder económico, que se sitúan más allá del poder político y tienden a mantenernos divididos. Acusan a los medios de partidismo -algo menos a la prensa escrita- y a la falta de neutralidad de los periodistas, aun cuando se confía más en su neutralidad, y vienen a coincidir con los más jóvenes en que uno de los objetivos de la manipulación de la información es inducir aquellos asuntos de los que se quiere que se hable.

La persuasión sobre el partidismo de los medios, como supuesto de partida, viene a explicar la pauta habitual de comportamiento en la recogida de información: informarse a través de varios medios. En este sentido, la elección de los medios viene a fundamentarse principalmente en dos criterios: la valoración global del medio en su conjunto y la empatía suscitada con quien transmite la información, algo que parece tener una especial incidencia entre las mujeres.

El segundo objetivo de análisis cualitativo fue indagar sobre las evocaciones espontáneas que le sugería a nuestro público informante el concepto de reputación mediática que, genéricamente, y sin diferencia de franjas de edad, se identificó con la noción de credibilidad -tanto de los medios como de los periodistas-y que, en un primer nivel se asocia con otros dos atributos subordinados: el rigor y la objetividad informativas.

Además de los atributos citados que espontáneamente son asociados a la reputación mediática, en el discurso social, en su conjunto, también se identifica con la financiación de los medios y los intereses empresariales que tienen detrás, con la trayectoria del medio en cuanto a la calidad de la información -destacando la exigible diferencia entre información y opinión y el reconocimiento del error como un criterio reputacional- y la formación de sus profesionales en cuanto a la adaptación a la digitalización. Además, se da paso también al intercambio de opiniones sobre el contraste de las informaciones y la relevancia de las fuentes; se señala la importancia de la pluralidad, sobre todo de cara a las generaciones actuales, y la necesidad de dar cabida al debate dentro del propio medio y no tener que acudir a otro medio para conocer otras opiniones. Finalmente, entre las evocaciones espontáneas citadas, la reputación mediática está en la actualidad en crisis por la rapidez de los acontecimientos y el exceso de información al que las audiencias están expuestas, lo que -se entiende- va en detrimento del necesario contraste de la información. En este sentido, los requisitos exigibles al contraste de una información aparecen también en el discurso y los podemos concretar en cuatro: el uso de documentos y fuentes fiables; la calidad de la investigación; la capacidad de crear debate y el reconocimiento de los errores.

El tercer objetivo de la indagación cualitativa exigía ahondar en el tratamiento del concepto de la reputación mediática y, en tal sentido, cuando el discurso de nuestros/as informantes avanza en el tratamiento de la reputación de los medios, recoge los aspectos señalados en la evocación espontánea anterior y aporta otros ampliando el desarrollo de las lógicas argumentales en las que sustentan sus opiniones:

- la trayectoria seguida por el medio y por quién lo dirige, y sus relaciones con el entorno institucional;

- la profesionalidad de los periodistas y de quienes intervienen en los programas de información;

- la veracidad de la información y el rigor a la hora de exponerla;

- la coherencia y constancia en los ideales que dice representar el medio y, por último,

- la opinión de las personas del entorno personal del informante a quienes se atribuye una mayor experiencia. 
Llegados a este punto conviene recapitular los hallazgos cualitativos obtenidos los cuales constituirían tres condiciones de la reputación mediática según la población informada: la credibilidad del medio, que exige independencia; la objetividad de la noticia que requiere imparcialidad, y la diversidad de fuentes siempre que estén suficientemente contrastadas.

Una vez establecidos los primeros límites al concepto de reputación mediática, y teniéndolos muy presentes, se interpeló directamente a nuestros informantes sobre la reputación de los medios que habitualmente consumían ellos. La conclusión fue clara: no cabe confiar en la reputación actual de los medios.

Las causas de esta valoración son diversas: se proyecta sobre los medios la crítica y la falta de reputación que envuelve actualmente a otras muchas instituciones; aun cuando se llega a reconocer que hay medios con más reputación que otros, sobre todo entre los nuevos, se parte del estereotipo de una falta de reputación generalizada; se piensa que los propios medios han contribuido a ello por la manipulación constante, aun cuando también haya influido el momento de confusión en que vivimos y la rapidez con la que se suceden las noticias. Incluso la crítica a la mala reputación de los medios acaba revertiendo también hacia los propios consumidores de la información que reconocen

"seguir leyendo a periodistas sancionados por mentirosos"

o que dicen

"buscar el rigor, pero quedan atrapados por el morbo".

En la última fase del análisis cualitativo se intentó que nuestro público informante identificara las variables de las que, según su parecer, dependía la reputación mediática. En este sentido, la credibilidad opera como el concepto matriz de la construcción social de la reputación de los medios: es el primer significante del que echa mano el discurso de ambos grupos de edad en la primera evocación espontánea sobre el tema; aparece como referente permanente cuando se avanza en el tratamiento de los factores y condiciones que garantizan la reputación y, por último, se sitúa en una posición diferente al resto de las variables como condición previa o resultado de las mismas, o como condición final para una buena reputación mediática.

Para la población informada española la credibilidad de los periodistas se relaciona básicamente con el rigor, la pluralidad y la imparcialidad; la credibilidad del medio se asocia básicamente con su trayectoria y su capacidad de rectificar. El rigor es algo que se prueba noticia a noticia, la credibilidad se gana con el transcurso del tiempo. El rigor acaba decantándose como una atribución personal a la profesionalidad del periodista, como una actitud en el tratamiento de la noticia; una actitud de trabajo del profesional que se asocia con: seriedad, trabajo y esfuerzo; exigencia con uno mismo, constancia, coherencia, contraste de las informaciones y pluralidad en cuanto a las opiniones.

En el discurso cualitativo analizado, rigor se asocia a objetividad. La objetividad es una cualidad que se atribuye sobre todo a la información y se argumenta acerca de la misma según la relación que se establece entre la información transmitida y la opinión de quien lo hace. De hecho se habla de objetividad en un doble sentido: uno más literal -dar la información tal como es, sin mezclarla con la propia opinión, absteniéndose de ella- y otro sentido más abierto, dar información con un abanico de opiniones acerca de la misma. En este contexto vuelve a plantearse uno de los temas recurrentes en el discurso cualitativo sobre la reputación mediática, que presenta una permanente división de opiniones entre quienes siguen manteniendo que la objetividad ha de basarse en la información pura y dura, sin quedar contaminada por la opinión, y los que creen que de ser así no cabe hablar de objetividad porque todos buscamos opinión.

Ante la pregunta, introducida en el debate del estudio cualitativo: ¿cabe ser objetivo? Los informantes de más edad opinan, de forma significativa, que en los medios se ha perdido objetividad en las dos últimas décadas. Antes -se afirma- los periodistas eran más independientes y más creíbles, ahora se han hecho más partidistas y su opinión está cada vez más sesgada. Como en el caso de la credibilidad y de la reputación, se acaba reconociendo que hay medios más objetivos que otros, en tanto en cuanto no aparecen abiertamente posicionados en un lado o en otro y, de nuevo, vuelve a revertirse parte de la responsabilidad de que no haya más objetividad sobre los propios consumidores de los medios denunciándose una actitud ambivalente entre querer la objetividad y mantener las mentiras; o pretender una información objetiva y permitir una prensa amarilla que alimenta el morbo de las cosas, bajo el pretexto de tener opinión propia. En conclusión, el intercambio de opiniones pone de manifiesto la lógica latente en la que se sustenta dicha ambivalencia: por una parte algunos se aferran a la exigencia de separar información de opinión para denunciar la falta de objetividad de los periodistas y de los medios y por otra -redundante- se ve muy difícil separar información de opinión porque todos buscan crear opinión cuyo efecto cercena la objetividad.

Otra asociación clara que descubre el discurso cualitativo es el binomio imparcialidad e independencia, sobre el que nuestros informantes aunque argumentan conjuntamente sobre ambos conceptos, sin embargo el intercambio dinámico de opiniones acaba estableciendo una clara diferenciación entre los mismos: la imparcialidad es una cualidad que se demanda en contraposición al partidismo como una cualidad subjetiva que se atribuye a los periodistas 
"no decantarse por un color u otro"; "no dar una noticia para favorecer a unos o a otros"; "no tomar parte por la noticia".

Por el contrario, la independencia se diferencia de la imparcialidad

"que no te digan las noticias que tienes que dar"; "que te dejen dar la noticia como es".

Pero como en el debate sobre la objetividad, también aquí surge la pregunta ¿se puede hablar de la independencia de los medios? La respuesta encuentra un cierto grado de consenso en el discurso analizado:

"Solo cabe hablar de una independencia relativa, en la medida en que todos dependemos del dinero para sobrevivir y nadie muerde la mano de la que come y los grupos de poder se valen cada vez más de la información manipulada en su beneficio".

La dependencia a la que se atribuye mayor poder sobre la actuación de los medios es la dependencia económica. Un poder que se ejerce supuestamente a través de la financiación y la publicidad. De cualquier forma se tiene en cuenta que quien dirige el medio, y sus profesionales, han de velar también por la reputación del mismo procurando contrarrestar en alguna medida su dependencia económica e incluso sus afinidades ideológicas.

La siguiente etapa metodológica fue la definición del universo mediático y la selección de la muestra de análisis en España, que se basó en una doble indagación de naturaleza cuali-cuantitativa: 22 entrevistas en profundidad a profesionales con un mínimo de 15-20 años de experiencia en medios de referencia y una encuesta por cuestionario a 135 profesionales de medios, dircoms de grandes empresas y líderes de opinión.

Se partió inicialmente de cuatro tipos de medios: prensa en papel, medios nativos digitales, radio y televisión, mencionados al menos una vez: un total de 19 medios.

De ellos fueron excluidos los tres diarios Expansión, Cinco días y Marca por ser de prensa especializada, cerrando la muestra de análisis con 16 medios: 5 cabeceras de prensa escrita generalista; 4 radios; 4 televisiones y 3 diarios nativos digitales. Estos son los 16 medios con mejor reputación en España, ordenados alfabéticamente:

1. $A B C$

2. Antena 3

3. COPE

4. El confidencial

5. El diario.es

6. El mundo

7. El país

8. El periódico de Cataluña

9. InfoLibre

10. La sexta

11. La vanguardia

12. Onda cero

13. $R N E$

14. SER

15. Telecinco

16. TVE

Una vez seleccionada la muestra de análisis se procedió a dar respuesta a las preguntas de investigación planteadas, la primera de las cuales se refería a los valores que determinan la reputación de un medio de comunicación.

En la escasa doctrina empírica existente sobre la reputación mediática Ortiz-de-Guinea-Ayala (2015) y Ortiz-de-Guinea-Ayala, Villafañe y Caffarel (2017) concluyen que hay dos valores de reputación canónicos: los profesionales o periodísticos y los empresariales. En el estudio cualitativo efectuado en esta investigación a profesionales de los medios de comunicación con idéntico objeto - descubrir qué valores o dimensiones básicas determinaban la reputación de los medios- el discurso fue muy redundante respecto a las variables que ya habían aparecido en el discurso de la población informada: credibilidad, independencia, rigor, transparencia en la gestión, independencia económica... Se les planteó entonces a los expertos, como ejemplo de lo que podría ser un valor mediático de carácter general, los ya citados valores periodísticos y empresariales, invitándoles además a añadir otros valores, de cualquier naturaleza, pero de similar amplitud que los dos citados. Se mencionaron 10 valores diferentes de los que, según nuestros informantes, dependería la reputación mediática, obteniéndose un total de 36 menciones de esta decena de valores: periodísticos, empresariales, democráticos, tecnológicos, axiológicos, relación con los poderes fácticos, reputación personal de los responsables del medio, transparencia, relación con la audiencia. 
La segunda pregunta de la investigación -el peso o importancia de cada uno de los valores canónicos de la reputación mediática- ofreció resultados contundentes. Los valores periodísticos fueron mencionados en 15 ocasiones, un $41,7 \%$ del total; los empresariales 11 veces, un 30,6\%; los democráticos obtuvieron 2 citas, igual que los tecnológicos, un 5,5\% respectivamente, mientras que los otros seis valores fueron señalados tan solo en una ocasión.

Dada la concentración de las menciones se tomó la decisión de adoptar los valores periodísticos y empresariales como los valores canónicos de la reputación mediática y, para cuantificar el peso de cada uno de ellos, procedimos a re-ponderar en base 100 todas las menciones considerando únicamente los dos valores canónicos confirmados, obteniendo como resultado que los valores periodísticos tienen un peso del 57,7\% en la reputación mediática y los empresariales un 42,3\%.

La tercera pregunta de investigación exigía jerarquizar los grupos de interés o stakeholders de los medios de comunicación en función de su importancia para la reputación de dichos medios. El público informante fue, de nuevo, la muestra cualitativa de directores, editores y profesionales de los medios y dircoms entrevistados. Estos son los grupos de interés, con 10 o más menciones, en importancia decreciente:

- Audiencia (21 menciones)

- Anunciantes (20)

- Poder económico (17)

- Profesionales de los medios (16)

- Sociedad (13)

- Accionistas (12)

- Poder político (10)

La cuarta pregunta de investigación se refería a las variables o factores determinantes de estos dos valores, es decir, qué variables -periodísticas y empresariales- construyen los valores de la reputación mediática. Para la selección de las decenas de atributos que los profesionales de los medios y directores de comunicación de grandes corporaciones mencionaron como dependientes y determinantes de los valores periodísticos y empresariales de la reputación de un medio de comunicación, se utilizó la técnica MaxDiff a partir de cuyos resultados se obtuvieron las siguientes diez variables, de las que las 6 primeras tienen carácter periodístico y las 4 últimas son las variables empresariales.

- Credibilidad.

- Rigor informativo.

- Calidad periodística.

- Independencia.

- Ética y deontología profesional.

- Contribución a la democracia.

- Sostenibilidad e independencia económica.

- Transparencia en la gestión.

- Responsabilidad de la propiedad.

- Derechos y deberes de los trabajadores.

Para la identificación de estas variables de reputación mediática en la muestra de población informada se llevó a cabo un análisis Shapley Value Regression que permitió identificar y jerarquizar cuáles de estas variables tienen una mayor influencia en la reputación global de un medio entre todas las que surgieron durante el estudio cualitativo con la citada población informada, y cuáles tienen menos.

Esta importancia puede contribuir tanto en sentido positivo: cuanto mejor valoración de una variable de reputación, mejor reputación global, como en sentido inverso, detectándose aspectos en los que se recogen valoraciones bajas que contribuyen a una menor reputación en conjunto.

Se ha escogido, en este caso, realizar una regresión Shapley Value, dada la alta correlación de algunas de las variables independientes con las otras -los valores del VIF, Variance Inflaction Factor, de las variables independientes están entre 3,7 y 7,2, siendo la regla estándar, según Hair et al. (2010), que un VIF > 4 denota problemas de multicolinealidad-. Si realizáramos una regresión lineal por mínimos cuadrados ordinarios, en este caso en el que varias de las variables independientes están altamente correlacionadas entre sí, no seríamos capaces de discriminar el efecto de cada variable por separado, ya que hay cierta varianza que está compartida entre variables, y el modelo no nos permitiría determinar a cuál de las variables pertenece. El modelo Shapley Value resuelve este problema, ya que hace múltiples cálculos para los diferentes valores, incluso la ausencia, de cada una de las variables independientes, pudiendo aislar su efecto y determinar con certeza cuánto explica cada variable independiente de la dependiente. Sabiendo esto, podemos jerarquizarlas en su orden de importancia explicativa.

Gracias al análisis efectuado, y de forma general, e independientemente del tipo de medio, no solo se han podido identificar las variables mediáticas que más afectan a la reputación de un medio, según la población informada, sino que también se han jerarquizado por cada tipo de medio y para cada medio independientemente. Las siete variables mediáticas para la población informada en España son: 


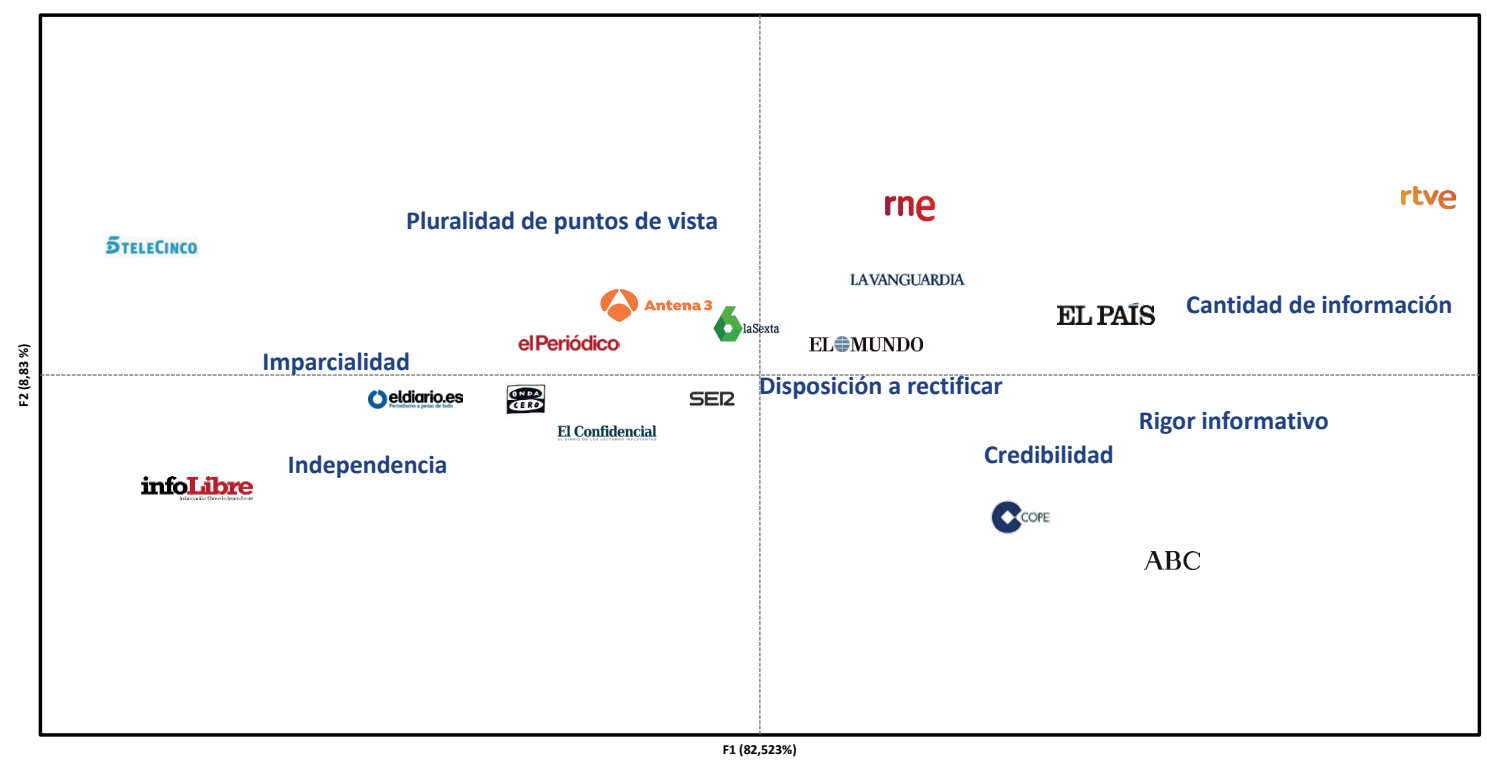

Gráfico 1. Medios y variables de reputación mediática en función de las cuales el público informante ha efectuado su evaluación. Realizado mediante Análisis Factorial de Correspondencias. Base: Total muestra población informada (1.000 casos)

- La disposición a rectificar.

- La credibilidad.

- El rigor informativo.

- La cantidad de información proporcionada.

- La pluralidad de puntos de vista.

- La independencia.

- La imparcialidad.

En el gráfico 1 se muestra un mapa de posicionamiento en el que se representan -según la valoración de la población informada consultada- los medios de la muestra y las variables de reputación mediática, en función de las cuales este público informante ha efectuado su evaluación.

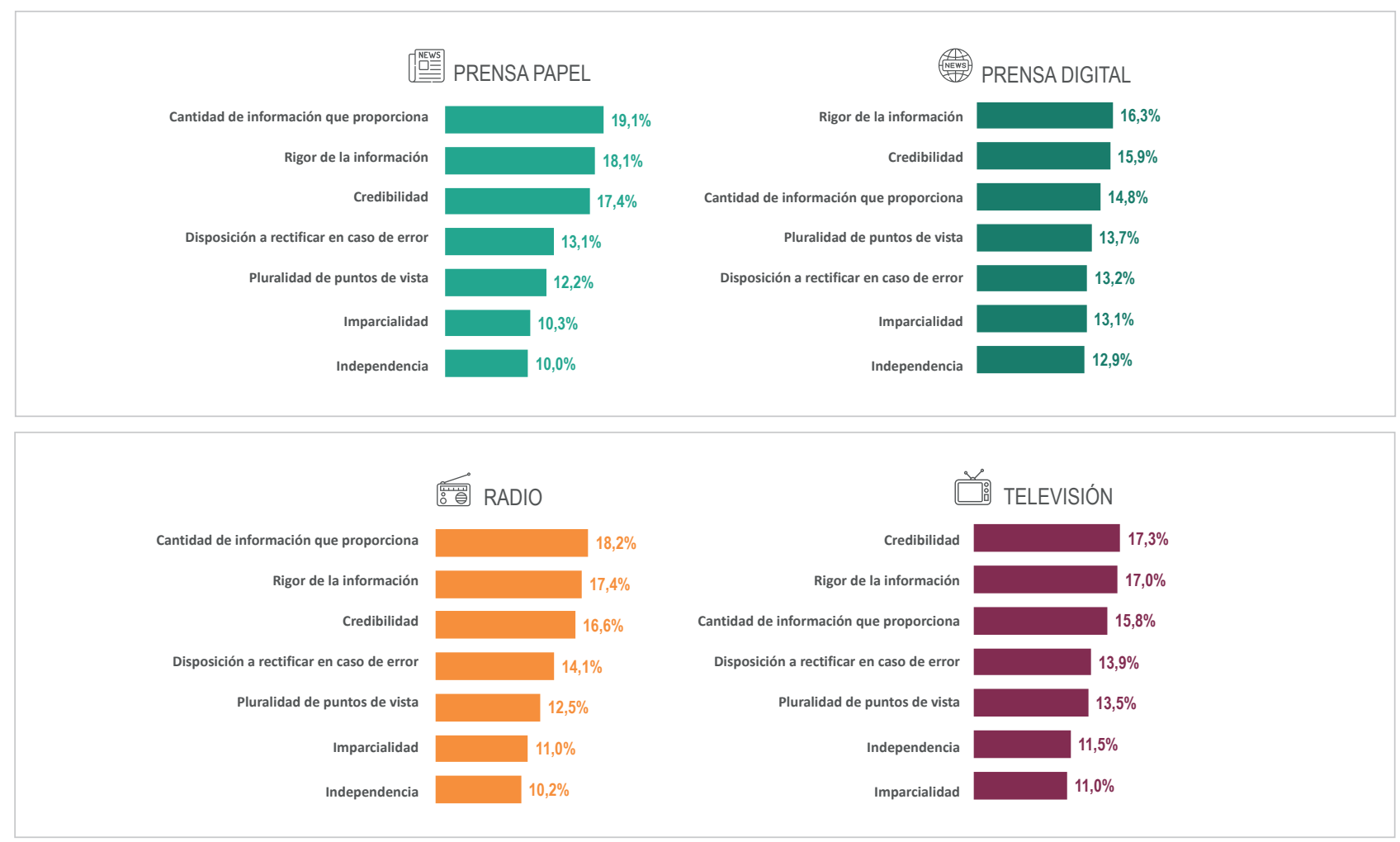

Gráfico 2. Jerarquización de las variables de reputación mediática para los cuatro tipos de medios analizados, efectuada por la población informada 


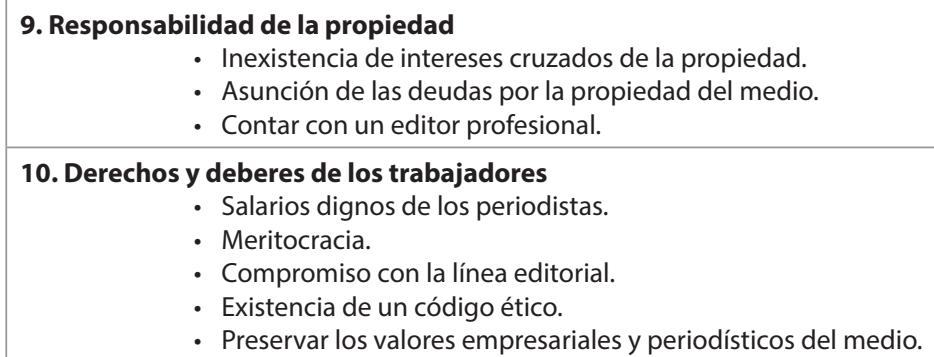

La sexta pregunta de la investigación planteaba la evaluación cuantitativa de la reputación mediática de los cuatro tipos de medios que configuran la muestra y, para evitar una correlación excesiva entre las variables e indicadores de reputación utilizadas hasta entonces se planteó en ambas muestras de análisis la siguiente pregunta, sin mencionar en ningún caso el término reputación mediática:

"A nivel general, y sin referirse a ningún medio concreto, ¿qué tipo de medio, diferenciando entre prensa en papel, prensa digital, radio y televisión, le parece a usted que tiene...?: más credibilidad, más rigor informativo, mayor imparcialidad, más predisposición a rectificar".

A partir de aquí exponemos las valoraciones de las dos muestras con las que hemos trabajado -profesionales de los medios y población informada- cuyas bases fueron de 135 y 1.000 casos respectivamente.

Para los profesionales, la prensa en papel es el medio asociado en mayor medida a las cuatro preguntas formuladas, y en especial al que se le atribuye mayor rigor informativo (60\%) y credibilidad (56\%) (gráfico 3). El aspecto menos asociado a la prensa en papel, aunque superior al resto de medios, es la imparcialidad (24\%).

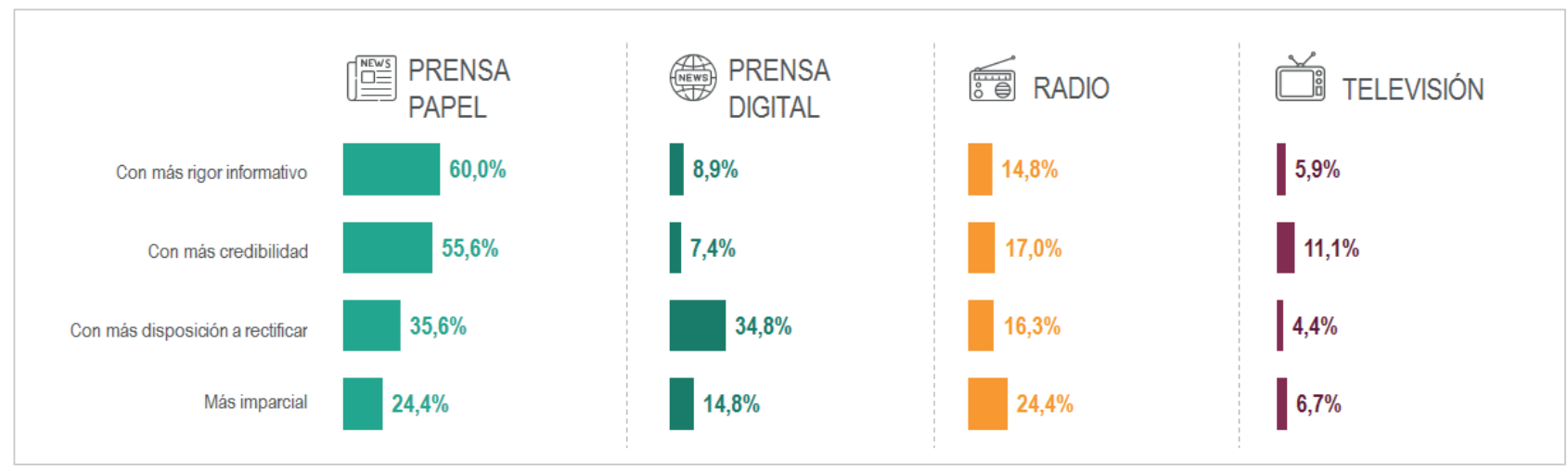

Gráfico 3. Valoraciones de los profesionales de los medios (135 respuestas)

La prensa nativa digital se asocia principalmente a la disposición a rectificar (35\%). La credibilidad es el aspecto menos vinculado a este tipo de medios (7\%). La radio es para los profesionales el medio más imparcial (24\%), pero también el de menor rigor informativo (15\%). La televisión es el medio peor valorado: solo un $4 \%$ piensa que tiene disposición a rectificar, estando su mejor valor asociado con la credibilidad (11\%), aunque por debajo de la prensa en papel y la radio.

Para la población informada (gráfico 4) también la prensa en papel es la más valorada en cuanto a su rigor informativo (32\%) y una mayor credibilidad (31\%). La imparcialidad es el aspecto menos asociado con este tipo de medio (23\%).

La prensa digital es la que tiene mayor disposición a rectificar (39\%), y el rigor de la información (24\%) y la credibilidad (25\%) son los aspectos menos asociados a estos medios. La radio es el medio más imparcial para un $36 \%$ de la población

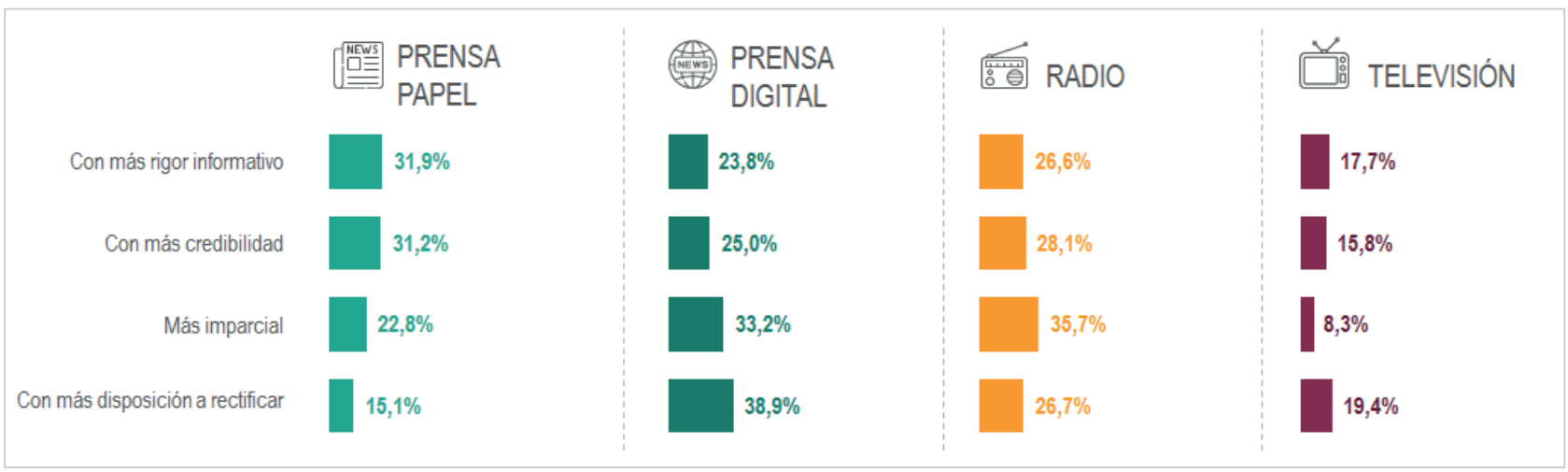

Gráfico 4. Valoraciones de la población informada (1.000 respuestas) 
informada. La televisión es el peor valorado en los cuatro factores evaluados, destacando únicamente en la disposición a rectificar (19\%) y en el rigor informativo (18\%), aunque por debajo del resto de medios en estos aspectos.

Una vez que se ha dado respuesta a las preguntas de investigación cuyos resultados permitieron evaluar la reputación de los medios que componen la muestra, recogemos en la tabla 1 la valoración que los profesionales han hecho sobre las 10 variables de reputación mediática de los ocho medios de prensa en papel: las 6 primeras correspondientes al valor reputación periodística y las 4 últimas al de reputación empresarial.

Tabla 1. Valoración de los profesionales sobre las 10 variables de reputación mediática de los ocho medios de prensa escrita. Se destacan los valores superiores (recuadros en verde) e inferiores (en rojo) para cada medio.

\begin{tabular}{|c|c|c|c|c|c|c|c|c|}
\hline & EL PAÍS & ELDIARIO.ES & $\begin{array}{c}\text { LA } \\
\text { VANGUARDIA }\end{array}$ & INFOLIBRE & $A B C$ & $\begin{array}{c}\text { EL } \\
\text { CONFIDENCIAL }\end{array}$ & EL PERIÓDICO & EL MUNDO \\
\hline Credibilidad & 6,79 & 6,63 & 6,73 & 6,84 & 5,22 & 6,01 & 5,96 & 5,53 \\
\hline Rigor informativo & 6,83 & 6,7 & 6,83 & 6,93 & 5,52 & 6,17 & 6,23 & 5,57 \\
\hline Calidad periodística & 7,43 & 6,79 & 7,25 & 6,63 & 5,82 & 6,11 & 6,28 & 6,1 \\
\hline Independencia & 5,48 & 6,22 & 5,48 & 6,79 & 4,36 & 5,58 & 5,26 & 4,68 \\
\hline Ética y deontología profesional & 6,63 & 6,56 & 6,33 & 6,86 & 5,43 & 5,63 & 6,04 & 5,2 \\
\hline Contribución a la democracia & 7,38 & 6,77 & 6,53 & 6,67 & 5,81 & 5,89 & 6,3 & 5,84 \\
\hline Sostenibilidad económica & 5,09 & 6,19 & 5,66 & 5,29 & 4,72 & 5,91 & 4,05 & 4,33 \\
\hline $\begin{array}{r}\text { Transparencia en la gestión } \\
\text { empresarial }\end{array}$ & 5,12 & 6,48 & 4,97 & 7,05 & 5,17 & 5 & 4,19 & 4,39 \\
\hline Responsabilidad de la propiedad & 5,54 & 6,27 & 5,72 & 6,78 & 5,63 & 5,36 & 4,79 & 4,8 \\
\hline $\begin{array}{r}\text { Derechos y deberes de los } \\
\text { trabajadores }\end{array}$ & 6,53 & 6,47 & 6,41 & 6,68 & 5,56 & 5,72 & 4,58 & 5,3 \\
\hline
\end{tabular}

Como se evidencia en el gráfico 5, El país es el periódico mejor valorado a nivel global por parte de los profesionales del sector con una puntuación de 6,94. En segundo lugar se sitúa La vanguardia, 6,79 puntos, seguido por Eldiario.es con 6,52 puntos. El mundo con un 5,69 de valoración global queda en última posición.

Lo que resulta más destacable es, sin embargo, el tipo de variables -las empresariales- que penalizan a todos los medios salvo $A B C$, que es el único que no tiene en rojo ninguna de las cuatro variables mediáticas de naturaleza empresarial (tabla 1).

El país $(6,68)$ es también para la población informada el medio de prensa escrita con mejor reputación (tabla 2). A partir de esa única coincidencia con la valoración de los profesionales los resultados son muy distintos: le siguen $E$ I mundo $(6,58)$ y $A B C(6,54)$. En las últimas cuatro posiciones aparecen los tres diarios digitales de la muestra.

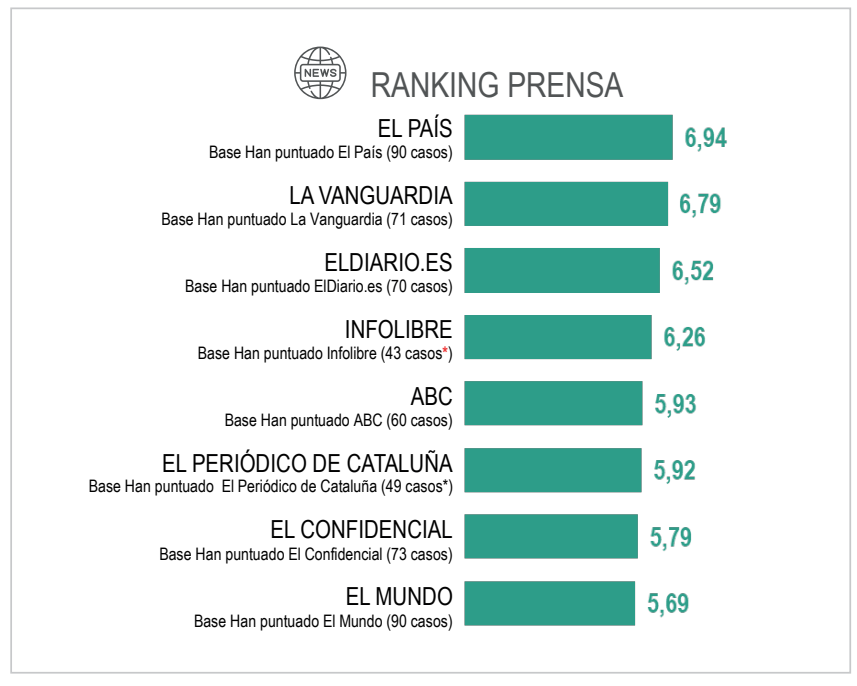

Gráfico 5. Valoración de los periódicos por parte de los profesionales del sector

Tabla 2. Valoración de la población informada sobre las 10 variables de reputación mediática de los ocho medios de prensa escrita. Se destacan los valores superiores (recuadros en verde) e inferiores (en rojo) para cada medio.

\begin{tabular}{|c|c|c|c|c|c|c|c|c|c|}
\hline & $\begin{array}{l}\text { TOTAL } \\
\text { PRENSA }\end{array}$ & EL PAÍS & EL MUNDO & $A B C$ & $\begin{array}{c}\text { LA } \\
\text { VANGUARDIA }\end{array}$ & $\begin{array}{l}\text { EL } \\
\text { CONFIDENCIAL }\end{array}$ & ELDIARIO.ES & EL PERIÓDICO & INFOLIBRE \\
\hline $\begin{array}{r}\text { Cantidad de información que } \\
\text { proporciona }\end{array}$ & 6,45 & 6,69 & 6,65 & 6,53 & 6,20 & 6,18 & 5,96 & 5,65 & 5,49 \\
\hline Rigor informativo & 6,20 & 6,23 & 6,38 & 6,25 & 5,94 & 5,99 & 5,78 & 5,48 & 5,55 \\
\hline Credibilidad & 6,18 & 6,21 & 6,32 & 6,24 & 5,86 & 5,98 & 5,75 & 5,47 & 5,62 \\
\hline $\begin{array}{r}\text { Disposición a rectificar en caso de } \\
\text { error }\end{array}$ & 5,97 & 5,91 & 6,06 & 5,91 & 5,70 & 5,90 & 5,77 & 5,39 & 5,56 \\
\hline Pluralidad de puntos de vista & 5,92 & 5,87 & 6,17 & 5,60 & 5,72 & 5,86 & 5,68 & 5,36 & 5,46 \\
\hline Independencia & 5,81 & 5,53 & 5,99 & 5,60 & 5,46 & 5,83 & 5,68 & 5,23 & 5,53 \\
\hline Imparcialidad & 5,74 & 5,58 & 5,82 & 5,48 & 5,51 & 5,74 & 5,62 & 5,24 & 5,56 \\
\hline
\end{tabular}


En cuanto a las variables mediáticas empleadas por la muestra de población informada existe una concentración extraordinaria ya que la cantidad de información que proporcionan es la variable más positivamente destacada por siete de los ocho medios y la imparcialidad más la independencia, las peor valoradas en último o penúltimo lugar por el mismo número y los mismos medios.

Otro de los factores que se analizó en la investigación es el grado de conocimiento de cada uno de los cuatro tipos de medios para comprobar si era posible establecer alguna correlación con la reputación mediática. La respuesta es parcialmente afirmativa, con la excepción de la prensa nativa digital en la que El confidencial es el más conocido, por un $54,8 \%$ de la muestra de profesionales quien, sin embargo, valora su reputación mediática $(5,79$ en base 10) por debajo de Eldiario.es -conocido por un $45,9 \%$ y con una reputación de 6,52- y de infoLibre $(21,5 \%$ y 6,26). En la prensa escrita en papel, radio y televisión sí coincide el medio más conocido y el de mejor reputación mediática. En la muestra de población informada, por el contrario, no existe apenas correlación entre conocimiento y reputación mediática.

Para la muestra de profesionales (tabla 3), la radio con mejor reputación mediática es la SER con un promedio de 6,88 puntos. Le siguen Onda cero $(6,32), \operatorname{RNE}(6,22)$ y $\operatorname{COPE}(5,06)$. Las dos primeras emisoras puntúan mejor en la variable calidad periodística; $R N E$ tiene su principal valoración en contribución a la democracia y la más baja en independencia. COPE obtiene la peor puntuación en independencia y la mejor en sostenibilidad económica.

Tabla 3. Valoración de los profesionales sobre las 10 variables de reputación mediática de 4 emisoras de radio

\begin{tabular}{|c|c|c|c|c|}
\hline & SER & ONDA CERO & RNE & COPE \\
\hline Credibilidad & 6,88 & 6,49 & 6,49 & 4,72 \\
\hline Rigor informativo & 6,82 & 6,39 & 6,42 & 4,89 \\
\hline Calidad periodística & 7,13 & 6,6 & 6,43 & 5,13 \\
\hline Independencia & 5,74 & 5,44 & 5,63 & 3,83 \\
\hline Ética y deontología profesional & 6,48 & 6,25 & 6,31 & 4,78 \\
\hline Contribución a la democracia & 7,04 & 6,37 & 6,59 & 5,02 \\
\hline Sostenibilidad económica & 5,64 & 6,04 & 6,05 & 5,37 \\
\hline $\begin{array}{r}\text { Transparencia en la gestión } \\
\text { empresarial }\end{array}$ & 5,11 & 5,33 & 5,81 & 4,57 \\
\hline Responsabilidad de la propiedad & 5,5 & 5,6 & 5,66 & 5,12 \\
\hline $\begin{array}{r}\text { Derechos y deberes de los } \\
\text { trabajadores }\end{array}$ & 5,8 & 5,7 & 6,56 & 5,29 \\
\hline
\end{tabular}

La radio con mejor reputación para la población informada es Onda cero $(6,82)$ (gráfico 6) cuya variable mejor valorada es la cantidad de información que proporciona y la peor valorada la imparcialidad (tabla 3). Le sigue la $\operatorname{SER}(6,81), \operatorname{RNE}(6,61)$ y $\operatorname{COPE}(6,50)$. También para este público las dos emisoras más reputadas -Onda cero y SER- lo son también en la variable calidad periodística. Igual coincidencia con la muestra anterior sucede con RNE -tiene su principal valoración en contribución a la democracia y la peor en su independencia- y con COPE cuya mejor puntuación es la sostenibilidad económica y la peor la independencia.

Tabla 4. Valoración de la población informada sobre las 10 variables de reputación mediática de 4 emisoras de radio. Se destacan los valores superiores (recuadros en verde) e inferiores (en rojo) para cada medio.

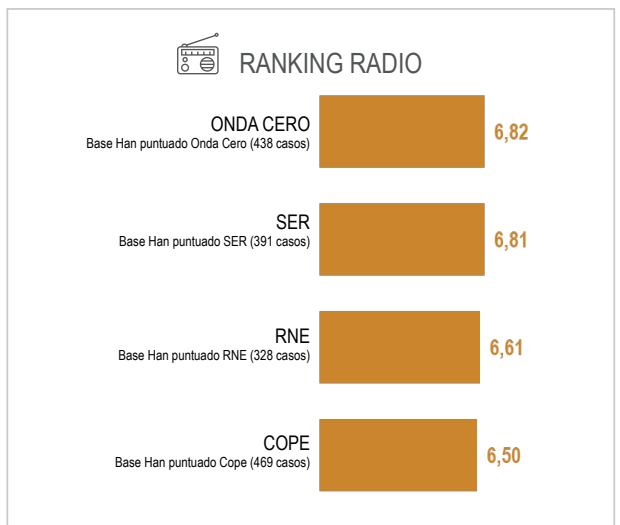

Gráfico 6. Reputación de las emisoras de radio en opinión de la población informada

\begin{tabular}{|c|c|c|c|c|c|}
\hline & $\begin{array}{l}\text { TOTAL } \\
\text { RADIO }\end{array}$ & SER & ONDA CERO & RNE & COPE \\
\hline $\begin{array}{r}\text { Cantidad de información que } \\
\text { proporciona }\end{array}$ & 6,66 & 6,69 & 6,77 & 6,66 & 6,44 \\
\hline Rigor informativo & 6,48 & 6,54 & 6,65 & 6,45 & 6,26 \\
\hline Credibilidad & 6,47 & 6,56 & 6,70 & 6,43 & 6,21 \\
\hline $\begin{array}{r}\text { Disposición a rectificar en caso de } \\
\text { error }\end{array}$ & 6,21 & 6,35 & 6,41 & 6,23 & 5,91 \\
\hline Pluralidad de puntos de vista & 6,17 & 6,32 & 6,56 & 6,31 & 5,71 \\
\hline Independencia & 6,01 & 6,14 & 6,42 & 5,87 & 5,68 \\
\hline Imparcialidad & 5,95 & 6,13 & 6,34 & 5,94 & 5,53 \\
\hline
\end{tabular}


La muestra de población ofrece datos que parecen objetivar una fuerte polarización de la audiencia radiofónica respecto a COPE ya que esta emisora, por decirlo de una manera poco académica, es la más querida y la más denostada. Si se observa la distribución de las valoraciones de las cuatro emisoras (gráfico 7), se puede observar como COPE, pese a ser la emisora con menor reputación mediática $(6,50)$, cuenta con el mayor apoyo de sus oyentes entre las cuatro emisoras: el $23,6 \%$ de la audiencia valoran su reputación global con las dos mayores puntuaciones 9 ó 10, el porcentaje más alto de las cuatro radios. Sucede lo mismo con el rechazo ya que alcanza el $17,7 \%$ de la audiencia, varios puntos por encima de sus competidoras.

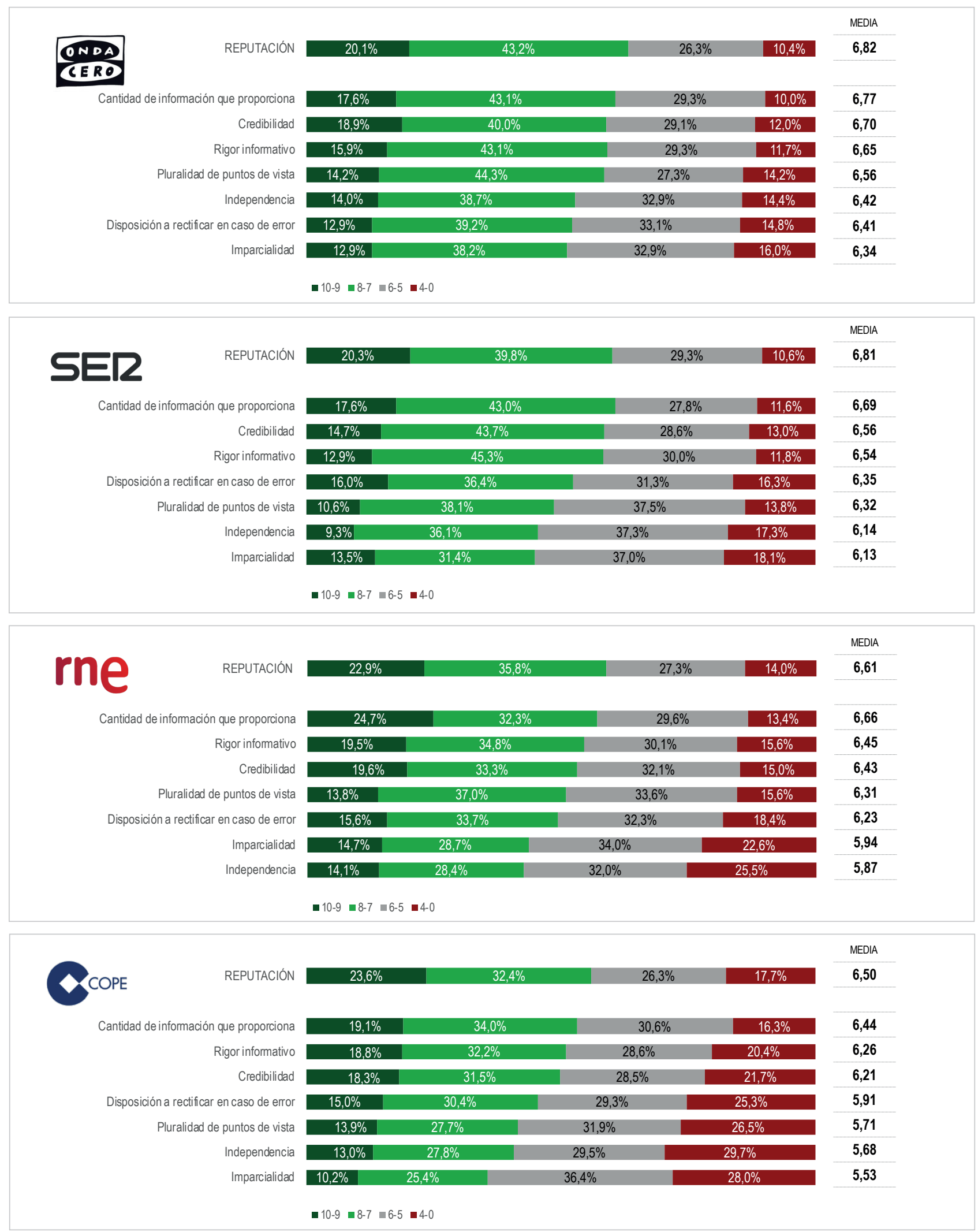

Gráfico 7. Distribución de las valoraciones de las cuatro emisoras analizadas 


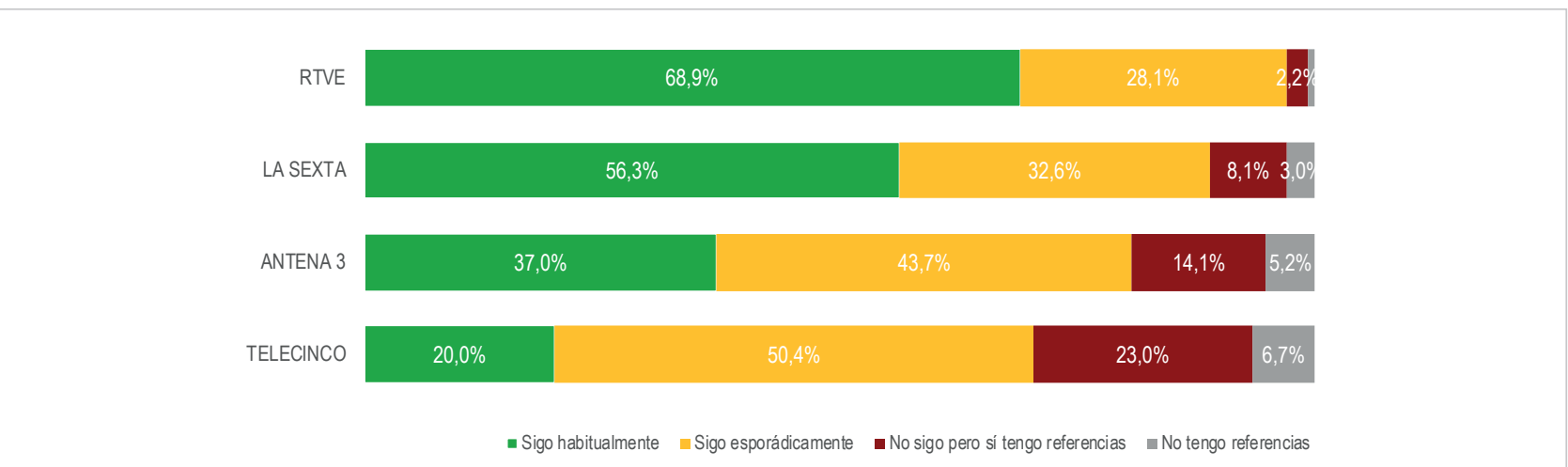

Gráfico 8. Cadenas de televisión más seguidas por la muestra de profesionales

La cadena de televisión más conocida, y seguida de manera habitual por la muestra de profesionales es TVE (69\%); la segunda posición es para La sexta $(56 \%$ y la tercera para Antena $3(37,0)$. La menos seguida es Telecinco, dado que un $30 \%$ de los profesionales entrevistados o no tiene referencias sobre este medio o no lo siguen (gráfico 8 ).

TVE es también la televisión más reconocida por su reputación $(6,40)$ entre la muestra de profesionales (gráfico 9). La sexta $(5,99)$, Antena $3(5,96)$ y Telecinco $(5,11)$ siguen a la televisión pública en cuanto a su reputación mediática (gráficos 10, 11 y 12). TVE, de igual manera que sucedía con $R N E$, tiene en la contribución a la democracia su variable más reconocida y en la independencia la peor.

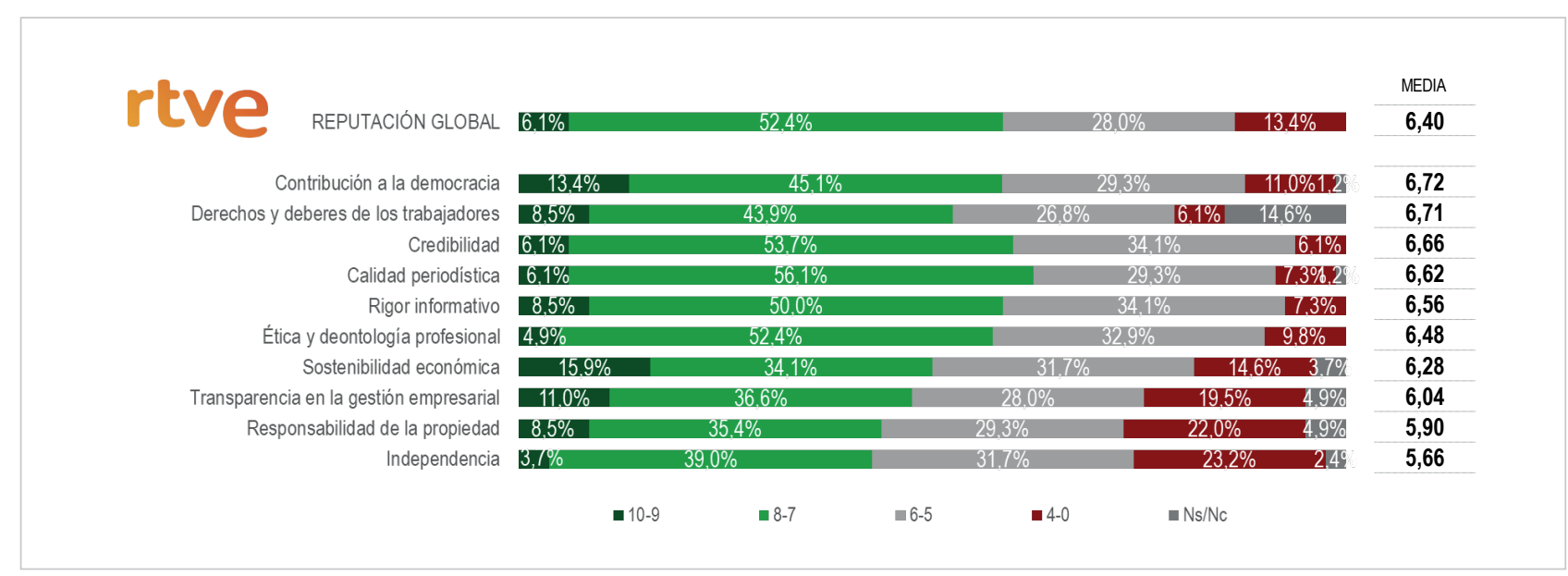

Gráfico 9. La contribución a la democracia $(6,72)$, los derechos y deberes de los trabajadores $(6,71)$ y la credibilidad del medio $(6,66)$ son los aspectos mejor valorados por los profesionales del sector sobre RTVE. La independencia del medio es el aspecto con una valoración más baja $(5,66)$.

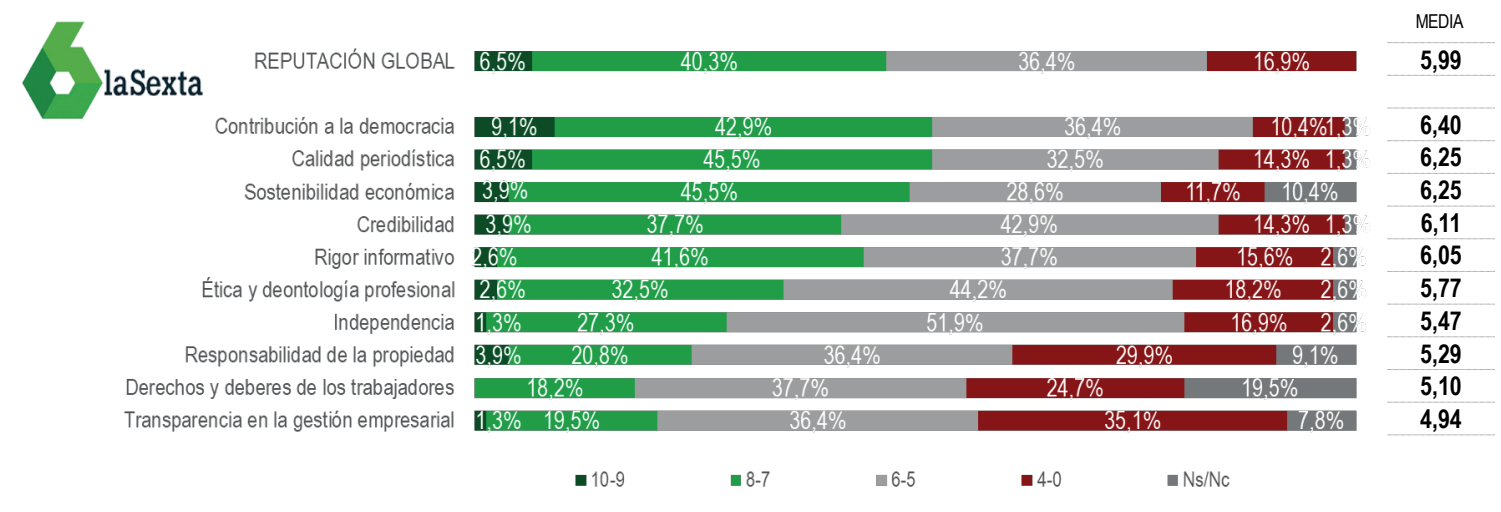

Gráfico 10. El aspecto mejor valorado de La sexta por los profesionales es la contribución a la democracia $(6,40)$. Es un medio menos asociado a la transparencia en la gestión empresarial, ya que ha obtenido la puntuación más baja $(4,94)$. 
Antena 3

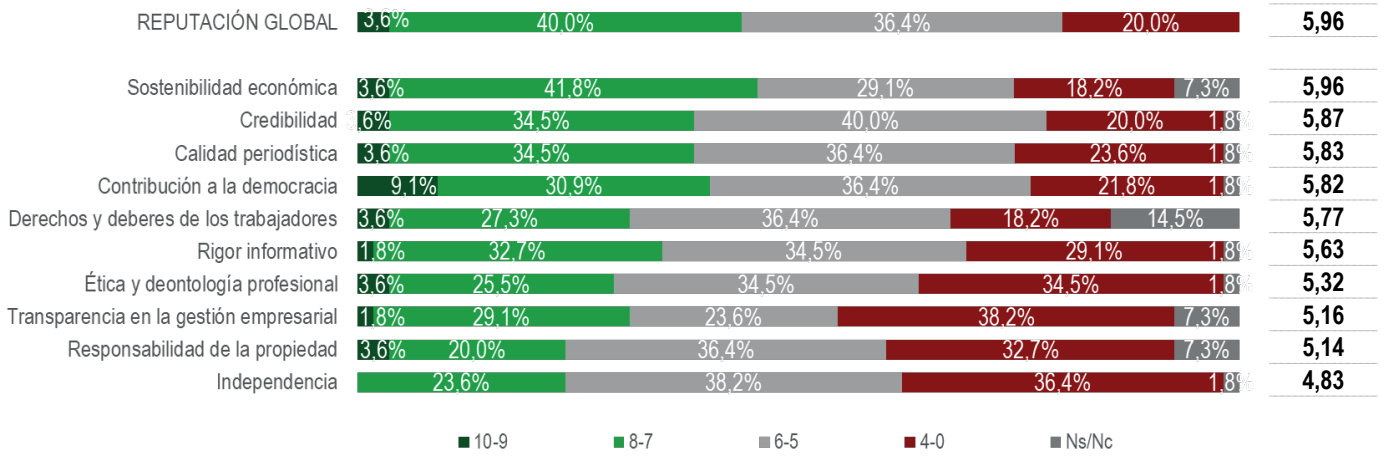

Gráfico 11. Los aspectos mejor valorados de la cadena de televisión Antena 3 son la sostenibilidad económica $(5,96)$, la credibilidad $(5,87)$ y la calidad periodística $(5,83)$. La independencia es el aspecto peor valorado $(4,83)$.

\section{TeleCinco}

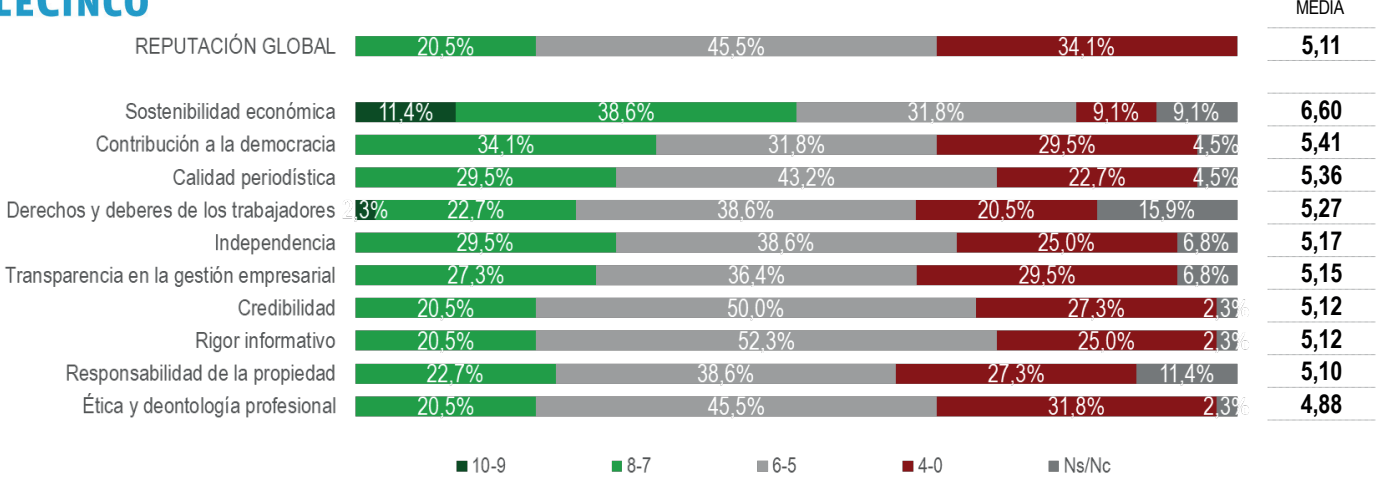

Gráfico 12. Telecinco es la cadena de televisión con la reputación global más baja. La sostenibilidad económica de la cadena es el aspecto mejor valorado $(6,60)$ y, por el contrario, la ética y deontología profesional es el peor valorado $(4,88)$, por debajo de los 5 puntos.

Tabla 5. Ranking de valoración de aspectos de cadenas de televisión (tabla I). Se destacan los valores superiores (recuadros en verde) e inferiores (en rojo) para cada medio.

\begin{tabular}{|c|c|c|c|c|}
\hline & RTVE & ANTENA 3 & LA SEXTA & TELECINCO \\
\hline Credibilidad & 6,66 & 5,87 & 6,11 & 5,12 \\
\hline Rigor informativo & 6,56 & 5,63 & 6,05 & 5,12 \\
\hline Calidad periodística & 6,62 & 5,83 & 6,25 & 5,36 \\
\hline Independencia & 5,66 & 4,83 & 5,47 & 5,17 \\
\hline Ética y deontología profesional & 6,48 & 5,31 & 5,77 & 4,88 \\
\hline Contribución a la democracia & 6,72 & 5,81 & \begin{tabular}{|l|l|}
6,39 \\
\end{tabular} & 5,4 \\
\hline Sostenibilidad económica & 6,28 & 5,96 & 6,25 & 6,6 \\
\hline $\begin{array}{r}\text { Transparencia en la gestión } \\
\text { empresarial }\end{array}$ & 6,04 & 5,16 & \begin{tabular}{|l|}
4,94 \\
\end{tabular} & 5,15 \\
\hline Responsabilidad de la propiedad & 5,9 & 5,14 & 5,29 & 5,1 \\
\hline $\begin{array}{r}\text { Derechos y deberes de los } \\
\text { trabajadores }\end{array}$ & 6,71 & 5,77 & 5,1 & 5,27 \\
\hline
\end{tabular}

Entre la muestra de población informada Antena 3 es el medio más conocido $(43,2 \%)$ y también el que cuenta con mejor reputación mediática $(6,54)$ (gráfico 13 ); seguida en cuanto a conocimiento, con un par de puntos de distancia entre cada una de las televisiones, por Telecinco $(40,3), \operatorname{TVE}(38,8)$ y La sexta $(36,5)$ (gráfico 12).

Las diferencias también son estrechas en cuanto a la reputación mediática de sus competidoras TVE $(6,36)$ y La Sexta $(6,21)$ y algo mayores - de un punto en base $10-$ en el caso de Telecinco $(5,51)$. Antena 3 tiene como variable más positiva 


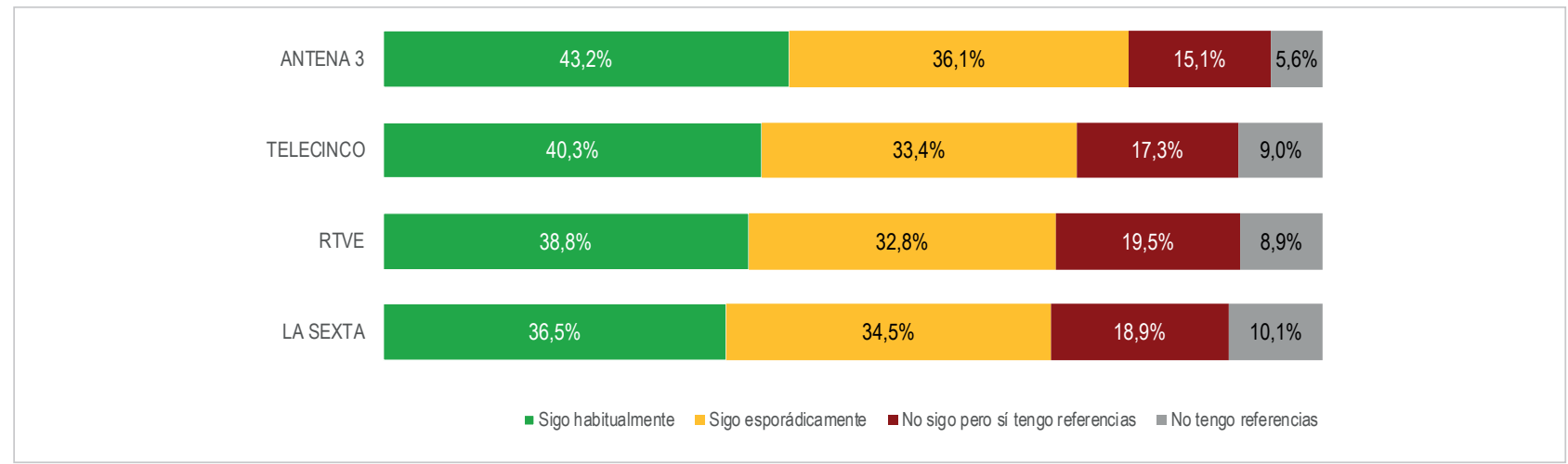

Gráfico 12. Conocimiento de las cadenas de televisión por parte de la población informada

Tabla 6. Valoración de diversos aspectos de las cadenas de televisión (tabla II)

\begin{tabular}{|c|c|c|c|c|c|}
\hline & $\begin{array}{l}\text { TOTAL } \\
\text { TV }\end{array}$ & ANTENA 3 & RTVE & LA SEXTA & TELECINCO \\
\hline $\begin{array}{r}\text { Cantidad de información que } \\
\text { proporciona }\end{array}$ & 6,38 & 6,56 & 6,60 & 6,56 & 5,78 \\
\hline Credibilidad & 6,05 & 6,24 & 6,10 & 6,27 & 5,54 \\
\hline Rigor informativo & 6,04 & 6,29 & 6,13 & 6,28 & 5,54 \\
\hline $\begin{array}{r}\text { Disposición a rectificar en caso de } \\
\text { error }\end{array}$ & 5,94 & 6,14 & 5,89 & 6,21 & 5,48 \\
\hline Pluralidad de puntos de vista & 5,92 & 6,17 & 5,71 & 6,18 & 5,64 \\
\hline Imparcialidad & 5,67 & 5,95 & 5,37 & 5,82 & 5,54 \\
\hline Independencia & 5,65 & 5,99 & 5,14 & 5,95 & 5,59 \\
\hline
\end{tabular}

la cantidad de información que proporciona $(6,56)$-la variable mejor puntuada también en sus tres competidoras- y en la imparcialidad la peor $(5,95)$.

\section{Conclusiones}

Dado lo prolijo de los resultados y conclusiones de la investigación, vamos a tratar de efectuar una síntesis de estas últimas. La primera de ellas, es la fragmentación del discurso sobre los medios o, por mejor decir, la multiplicidad de discursos sobre estos: el de los profesionales versus el de la población informada consumidora de información; dentro de esta, el de los jóvenes y el de los adultos; el doble discurso en torno al solapamiento de información y opinión...

El discurso social sobre los medios de comunicación se ha clausurado, inequívocamente, en torno a cuatro aspectos sobre los cuales la investigación ha ofrecido suficientes evidencias discursivas y empíricas y los investigadores hemos podido articular cuatro grandes conclusiones:

- Los medios de comunicación en España no poseen la suficien-

te reputación; muy al contrario, se los valora como instrumentos de manipulación al servicio, fundamentalmente, de las élites económicas.

- Los resultados de la investigación han permitido una conceptualización cabal de lo que se debe entender por reputación mediática y, también, la estratificación de esa reputación de los medios de comunicación en tres niveles de componentes: los valores de reputación, las variables dependientes de cada valor y, en un tercer nivel, los indicadores -que hagan posibles una evaluación empírica- de la reputación de cada medio.

- Las diferencias, en términos de reputación mediática, entre los diferentes tipos de medios a partir de una tipología que los divide en: medios de prensa escrita (5); medios nativos de prensa digital (3); radios (4) y televisiones (4). 
- Una evaluación pormenorizada de la reputación de cada uno de los 16 medios con mejor reputación en España que ha sido evaluados y la posibilidad de elaborar un ranking de reputación de los medios de comunicación en España abarcando, año a año, una muestra de medios mayor.

Respecto a la primera de las cuatro conclusiones existe un consenso amplio en que no existe confianza en los medios de comunicación por considerar que la información que difunden está manipulada. La existencia de una agenda mediática se valora negativamente por la población informada identificándola con el instrumento de las élites, principalmente económicas, pero también políticas, para primar unos contenidos noticiosos sobre otros en función de sus intereses y para alinear a la opinión pública con dichos intereses. Para esta muestra de público, esta falta de independencia de los medios es la manifestación más genuina de su falta de reputación.

De acuerdo con el comentario anterior acerca de la multiplicidad de discursos sobre los medios, ésta se pone de manifiesto con relación a la primera conclusión de la investigación. Existe una notable diferencia entre las conclusiones de los discursos que sobre los medios de comunicación tienen la población informada y los profesionales de la comunicación, los cuales también son bastante críticos, pero la diferencia de conocimiento sobre el funcionamiento de aquellos, su estructura empresarial, rutinas profesionales..., obviamente justifican esas diferencias de valoración, que en el ámbito de la reputación son fundamentales, ya que ésta se basa en el reconocimiento que hacen sus grupos de interés de su realidad y comportamiento mediáticos y el conocimiento de esta realidad exige una valoración informada.

Otra diferencia importante sobre la falta de reputación de los medios asociada a la manipulación de la información, asociada en este caso a la segmentación por edad, es la que se aprecia entre los grupos de edad joven -25-39 años- y el de mayor edad, 40-55 años. Todos ellos coinciden en su crítica a que los medios cuenten con una agenda noticiosa porque la identifican con el instrumento de la manipulación. Ante esta circunstancia la población informada, y sin diferencia aún entre el segmento joven y el adulto, persuadida de la manipulación de los medios por las élites se informa, o trata de hacerlo, acudiendo a diversos medios que seleccionan en función de dos criterios: la valoración global del medio versus el conjunto de todos los demás y, como segundo criterio de selección del medio, el o la periodista que difunde esa información, siendo las mujeres quienes mayor importancia atribuyen a este segundo criterio de selección mediática.

En lo que sí existe una diferencia apreciable entre los dos segmentos de edad de la población informada es en la fidelidad a los medios, tanto a los que siguen diariamente, como a los que utilizan coyunturalmente como medios de contraste o verificación de una noticia. En este sentido, la población joven es mucho más promiscua respecto a los medios y fuentes informativas consultadas: se informan poco en televisión y prensa escrita y, además, diferencian entre los sitios para informarse y aquellos para opinar.

En nuestra opinión el concepto de reputación mediática es uno de los hallazgos de esta investigación, tanto en lo que se deduce del discurso cualitativo como de su verificación empírica a partir del ulterior estudio cuantitativo. La reputación de un medio de comunicación es el resultado del reconocimiento que su audiencia hace de su comportamiento periodístico en la medida en que satisface sus expectativas respecto a su necesidad de información y a la identificación con el modo de obtenerla, editarla y difundirla de acuerdo, además, con un modelo empresarial justo y sostenible.

Es cierto que la anterior definición es en buena parte una definición aspiracional, pero los investigadores tenemos las evidencias suficientes que la justifican, especialmente aquellas que provienen de la muestra de población informada, la menos experta pero la que más aportó a una visión renovada de lo que deben ser los modos de hacer de los medios para recuperar la confianza y la reputación perdidos. Tomemos como ejemplo la principal conclusión de lo que la gente ilustrada, aunque no profesional, entiende por reputación de un medio: su credibilidad.

La credibilidad aparece en una posición diferenciada del resto de las variables de la reputación mediática al situarse como algo previo o resultante de las otras. De hecho, en la gran mayoría de las ocasiones, aparece seleccionada en primer lugar y en varios casos se destaca como consecuencia del resto de variables de la reputación de los medios, asociándose explícitamente con la reputación de dichos medios. La credibilidad de un medio aparece, en el discurso de la población informada, altamente correlacionada con otras seis variables de su reputación.

La credibilidad tiene una alta correlación con la trayectoria del medio -lo cual es absolutamente congruente con la teoría de la reputación- en la medida en que la reputación necesita tiempo para consolidarse como un valor estable de un medio o de cualquier organización. En este sentido, ambas variables -siempre desde el punto de vista de la población informada- se consolidan como la base de la matriz de sentido de la reputación mediática.

En el análisis cuantitativo de la relación entre los medios de la muestra y las variables de reputación mediática, según los profesionales y los dircoms, la credibilidad aparece (en rojo) también como el atributo central de la reputación de los medios tal como pone de manifiesto el mapa de posicionamiento de los medios en España (gráfico 14).

La tercera gran conclusión del estudio hace referencia a las diferencias de reputación mediática en función de la tipología de medios establecida: prensa escrita, nativa digital, radio y televisión. En este sentido, hemos dado preferencia a la muestra de profesionales de la comunicación, cuyos principales hallazgos sintetizamos seguidamente. 


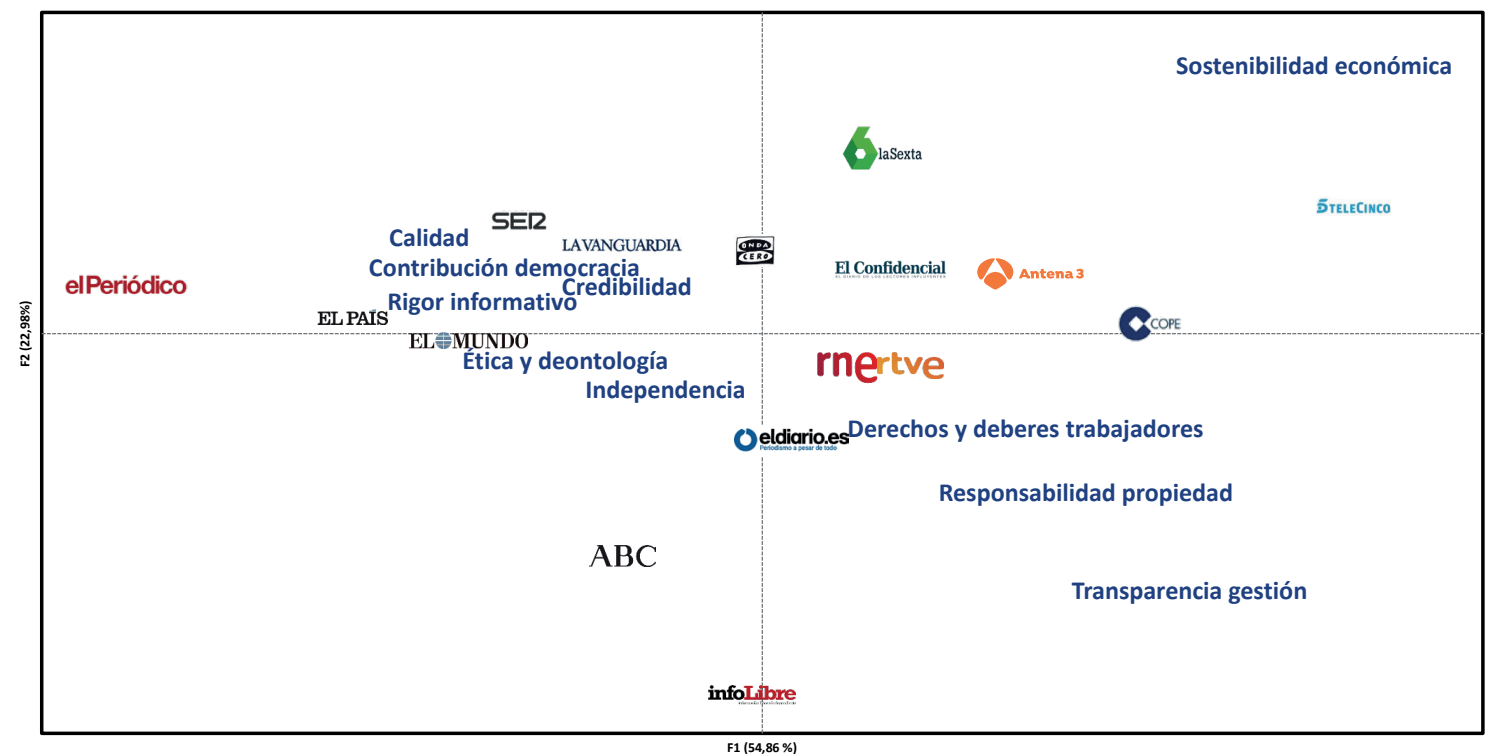

Gráfico 14. Asociación de aspectos a medios de televisión. Realizado mediante análisis factorial de correspondencias. Base: Total muestra de profesionales y dircoms (135 casos).

El país es el periódico mejor valorado a nivel global por parte de los profesionales del sector con una puntuación de 6,94. El segundo lugar es para La vanguardia $(6,79$ puntos), seguido por $A B C(5,93)$; El mundo $(5,69)$ ocupa la última posición entre los diarios en papel de la muestra. El diario digital con mejor reputación es Eldiario.es con 6,52 puntos, le siguen infoLibre $(6,26)$ y El confidencial $(5,79)$. La emisora que tiene una mejor reputación global es la SER, con una media de 6,88 puntos; a continuación se sitúan Onda cero $(6,32)$ y $R N E(6,22)$. La COPE $(5,06)$ es la peor valorada de las radios de la muestra. TVE es la cadena de televisión con mejor reputación para los profesionales. Por el contrario, Telecinco es la que peor reputación global registra entre este colectivo.

La última conclusión es la jerarquización de los 16 medios de la muestra reproducida a continuación en orden decreciente según su reputación mediática y de acuerdo a dos mediciones -la de la población informada y la de los profesionales de la comunicación- entre las que, como ya se ha justificado suficientemente, existen diferencias notables. Estos son los dos rankings de reputación de los medios de comunicación con mejor reputación en España. Esta afirmación final tiene mucha importancia porque en nuestro país existen decenas de medios y estos rankings recogen -desde dos grupos de informantes distintos- los medios más reputados de España.

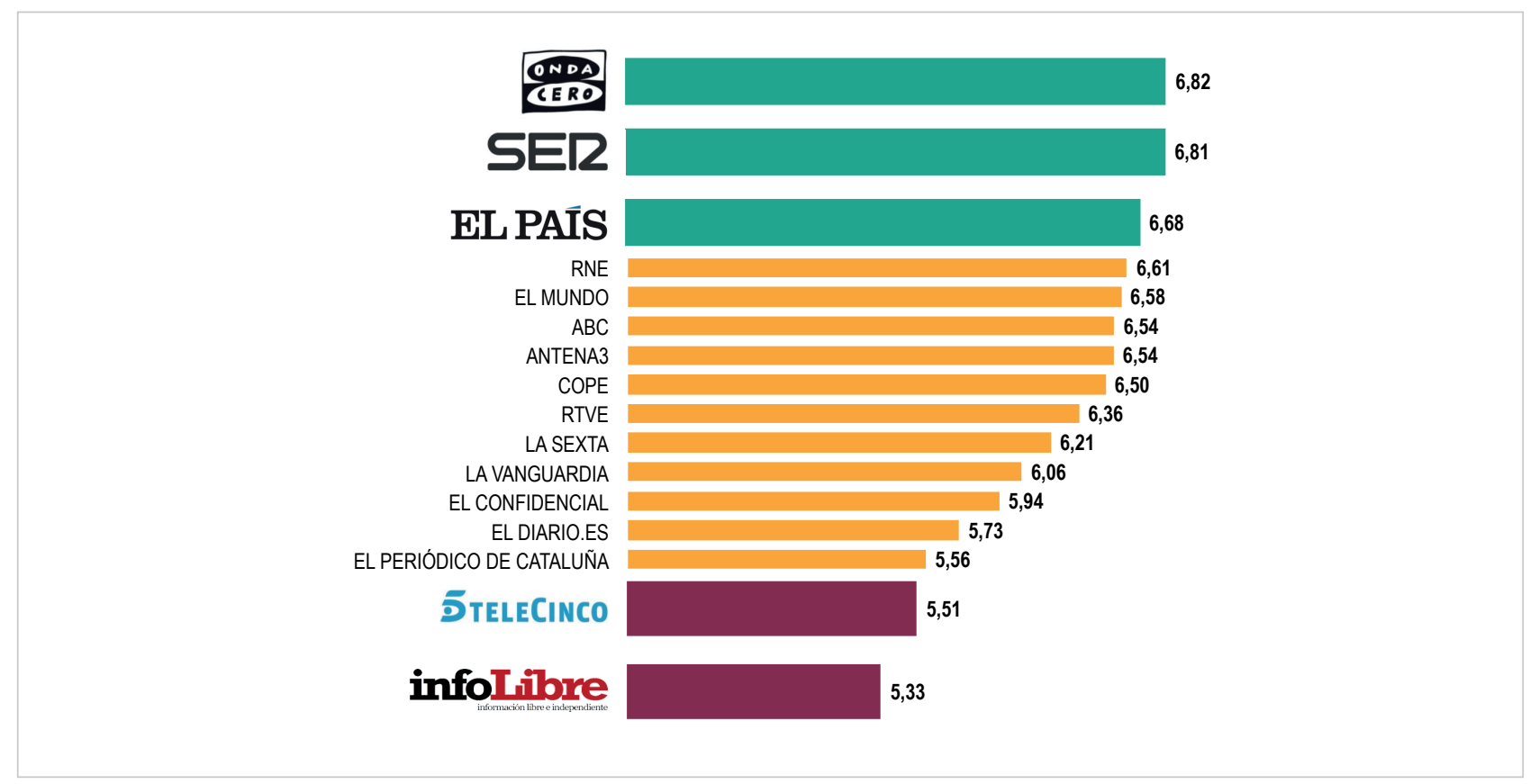

Gráfico 15. Ranking elaborado por Media REP Lab sobre los medios de comunicación con mejor reputación en España. Estudio por cuestionario a población informada: 1.000 casos. 


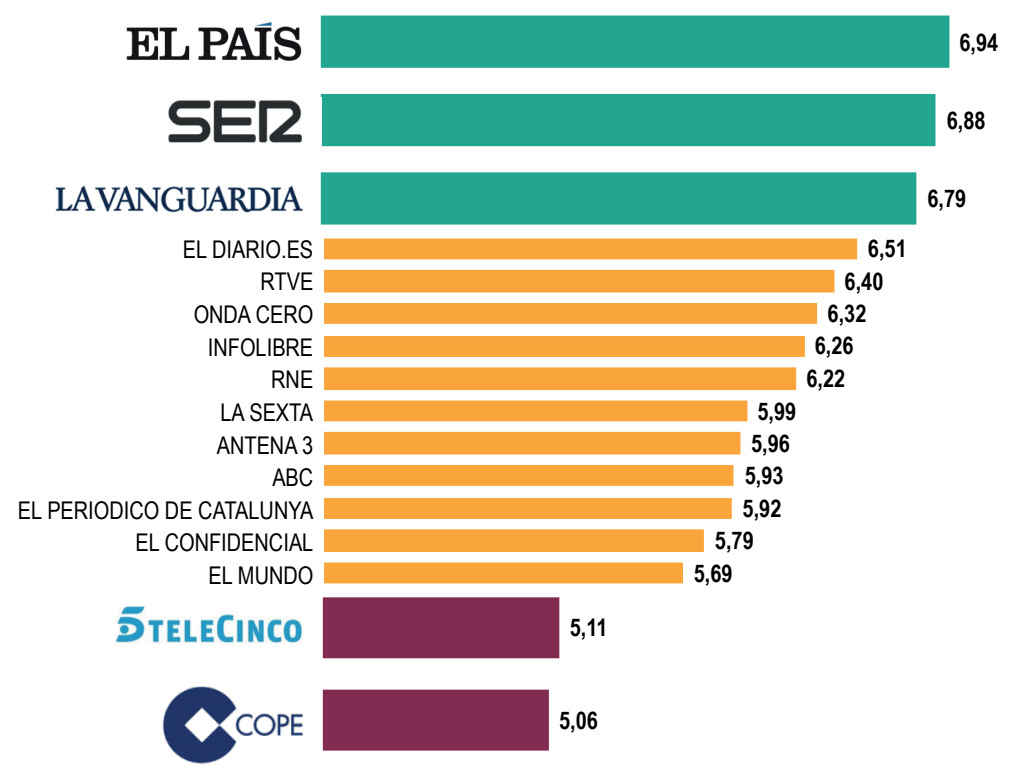

Gráfico 16. Ranking elaborado por Media REP Lab sobre los medios de comunicación con mejor reputación en España. Estudio por cuestionario a directores, editores, gestores, periodistas de medios y dircoms: 135 casos.

El equipo de académicos que ha realizado esta investigación se abstiene, premeditadamente, de elaborar conclusión alguna acerca de los resultados de ambos rankings, aunque como promotores del Media REP Lab (Media Reputation $L a b$ ) es nuestra intención seguir evaluando, y difundiendo, en el futuro la evolución de la reputación de los medios de comunicación en España y en Europa, con el mismo rigor con el que se ha efectuado el análisis contenido en este texto.

\section{Referencias}

Arregle, Jean-Luc; Hitt, Michael A.; Sirmon, David G.; Very, Philippe (2007). "The development of organizational social capital: attributes of family firms". Journal of management studies, v. 44, n. 1, pp. 73-95.

https://doi.org/10.1111/j.1467-6486.2007.00665.x

Aula, Pekka (2010). "Social media, reputation risk and ambient publicity management". Strategy and leadership, v. 38, n. 6, pp. 43-49.

https://doi.org/10.1108/10878571011088069

Berens, Guido; Van-Riel, Cees B. M. (2004). “Corporate associations in the academic literature: Three main streams of thought in the reputation measurement literature". Corporate reputation review, v. 7, n. 2, pp. 161-178.

https://doi.org/10.1057/palgrave.crr.1540218

Bunge, Mario (1985). La investigación científica. Barcelona: Ariel. ISBN: 9788435539128

Canavilhas, João (2011). “El nuevo ecosistema mediático”. Index. Comunicación, v. 1, n. 1, pp. 13-24.

https://journals.sfu.ca/indexcomunicacion/index.php/indexcomunicacion/article/view/4/7

Caruana, Albert; Chircop, Saviour (2000). "Measuring corporate reputation: a case example". Corporate reputation review, v. 3, n. 1, pp. 43-57.

https://doi.org/10.1057/palgrave.crr.1540098

Castelo, Manuel; Lima, Lúcia (2006). “Corporate social responsibility and resource-based perspectives”. Journal of business ethics, n. 69, pp. 111-132.

https://doi.org/10.1007/s10551-006-9071-z

Choi, Jaepil; Wang, Heli (2009). "Stakeholder relations and the persistence of corporate financial performance". Strategic management journal, v. 30, n. 8, pp. 895-907.

https://doi.org/10.1002/smj.759

Chun, Rosa (2005). "Corporate reputation: meaning and measurement". International journal of management reviews, v. 7, n. 2, pp. 91-109.

https://doi.org/10.1111/j.1468-2370.2005.00109.x

Cornell, Bradford; Shapiro, Alan C. (1987). “Corporate stakeholders and corporate finance". Financial management, v. 16, n. 1, pp. 4-14.

https://doi.org/10.2307/3665543 
Cravens, Karen; Goad, Elizabeth; Ramamoorti, Sridhar (2003). "The reputation index: measuring and managing corporate reputation". European management journal, v. 21, n. 2, pp. 201-212.

https://doi.org/10.1016/S0263-2373(03)00015-X

Davies, Gary; Chun, Rosa; Vinhas-da-Silva, Rui; Roper, Stuart (2015). Corporate reputation and competitiveness. Londres: Routledge. ISBN: 9780203218112

Deephouse, David L. (2000). "Media reputation as a strategic resource: an integration of mass communication and resource-based theories". Journal of management, v. 26, n. 6, pp. 1091-1112.

https://doi.org/10.1177/014920630002600602

De-la-Fuente-Sabaté, Juan-Manuel; De-Quevedo-Puente, Esther (2003). "The concept and measurement of corporate reputation: an application to Spanish financial intermediaries". Corporate reputation review, v. 5, n. 4, pp. $280-301$. https://doi.org/10.1057/palgrave.crr.1540180

Del-Fresno-García, Miguel (2011). “Cómo investigar la reputación online en los medios sociales de la web 2.0”. Cuadernos de comunicación Evoca, v. 5, n. 1, pp. 29-33.

https://www.researchgate.net/publication/277100601_Como_investigar_la_reputacion_online_en_los_medios_sociales_ de_la_web_20

Edelman Trust Barometer (2020). Edelman.

https://www.edelman.com/sites/g/files/aatuss191/files/2020-01/2020\%20Edelman\%20Trust\%20Barometer\%20 Global\%20Report_LIVE.pdf

Fombrun, Charles J. (1996). Reputation. Realizing value from the corporate image. Boston: Harvard Business School Press. ISBN: 0875846335

Fondevila-Gascón, Joan-Francesc; Del-Olmo-Arriaga, José-Luis; Bravo-Nieto, Vanesa (2012). “Presencia y reputación digital en 'social media': comparativa en el sector de la moda". Fonseca, journal of communication, n. 5, pp. 92-116. https://revistas.usal.es/index.php/2172-9077/article/view/12080

Hair, Joseph F.; Black, William C.; Babin, Barry J.; Anderson, Rolph E. (2010). Multivariate data analysis. London: Pearson. ISBN: 9780138132637

Helm, Sabrina (2007). "One reputation or many? Comparing stakeholders perceptions of corporate reputation". Corporate communications: an international journal, v. 12, n. 3, pp. 238-254.

https://doi.org/10.1108/13563280710776842

Hill, Charles W. L.; Jones, Thomas M. (1992). "Stakeholder-agency theory". Journal of management studies, v. 29, n. 2, pp. 131-154.

https://doi.org/10.1111/j.1467-6486.1992.tb00657.x

Informe anual de la profesión periodística (2015). Asociación de la Prensa de Madrid (APM). https://www.apmadrid.es/wp-content/uploads/2016/11/INFORME-PROFESION-APM-2015_baja_7M.pdf

Jensen, Michael C.; Meckling, William H. (1976). "Theory of the firm: managerial behavior, agency costs and ownership structure". Journal of financial economics, v. 3, n. 4, pp. 305-360.

https://doi.org/10.2139/ssrn.94043

Lange, Donald; Lee, Peggy M.; Dai, Ye (2011). “Organizational reputation: a review”. Journal of management, v. 37, n. 1, pp. 153-184.

https://doi.org/10.1177/0149206310390963

Luhmann, Niklas (2007). La realidad de los medios de masas. Barcelona: Anthropos Editorial. ISBN: 9788476588079

Madden, Mary; Smith, Aaron (2010). "Reputation management and social media”. Pew Research Center. https://www.pewresearch.org/internet/2010/05/26/reputation-management-and-social-media

Mahon, John F. (2002). “Corporate reputation: research agenda using strategy and stakeholder literature". Business and society, v. 41, n. 4, pp. 415-445. https://doi.org/10.1177/0007650302238776

Martín-de-Castro, Gregorio; Navas-López, José-Emilio; López-Sáez, Pedro (2006). “Business and social reputation: exploring the concept and main dimensions of corporate reputation". Journal of business ethics, v. 63, n. 4, pp. 361-370. https://doi.org/10.1007/s10551-005-3244-z

McWilliams, Abagail; Siegel, Donald S. (2001). "Corporate social responsibility: a theory of the firm perspective". The academy of management review, v. 26, n. 1, pp. 117-127.

https://doi.org/10.5465/amr.2001.4011987 
McWilliams, Abagail; Siegel, Donald S.; Wright, Patrick M. (2006). “Corporate social responsibility: strategic implications". Journal of management studies, v. 43, n. 1.

https://doi.org/10.1111/j.1467-6486.2006.00580.x

Metzger, Miriam J.; Flanagin, Andrew J.; Eyal, Keren; Lemus, Daisy R.; McCann, Robert M. (2003). "Credibility for the $21^{\text {st }}$ century: Integrating perspectives on source, message, and media credibility in the contemporary media environment". Communication yearbook, v. 27, pp. 293-335.

https://doi.org/10.1207/s15567419cy2701_10

Montero, Antonio; Forcadell, Francisco-Javier; Nájera, Juan-José (2011). "El ejercicio del liderazgo en los países Merco". En: Nogueira, J. M.; Villafañe, J. (eds.). La comunicación empresarial y la gestión de los intangibles en España y Latinoamérica. Madrid: Pearson, pp. 35-41. ISBN: 9788483227909

Murphy, Priscilla (2010). "The intractability of reputation: Media coverage as a complex system in the case of Martha Stewart". Journal of public relations research, v. 22, n. 2, pp. 209-237.

https://doi.org/10.1080/10627261003601648

Nahapiet, Janine; Ghoshal, Sumantra (1998). "Social capital, intellectual capital and the organizational advantage". Academy of management review, v. 23, n. 2, pp. 242-266.

https://doi.org/10.5465/amr.1998.533225

Nájera, Juan-José; Montero, Antonio; Forcadell, Francisco-Javier (2014). “Una propuesta de medida de la reputación corporativa en el ámbito empresarial basada en redes sociales". XXIII Congreso Nacional de Acede, Málaga.

Newell, Stephen J.; Goldsmith, Ronald E. (2001). "The development of a scale to measure perceived corporate credibility". Journal of business research, v. 52, pp. 235-247.

https://doi.org/10.1016/S0148-2963(99)00104-6

Ortiz-de-Guinea-Ayala, Yolanda (2015). El periodismo y la reputación de los periodistas en tiempos de crisis". Index. Comunicación, v. 5, n. 2, pp. 83-100.

https://journals.sfu.ca/indexcomunicacion/index.php/indexcomunicacion/article/view/167

Ortiz-de-Guinea-Ayala, Yolanda; Villafañe, Justo; Caffarel, Carmen (2018). "Investigación para la evaluación de la reputación de los medios de comunicación". Revista latina de comunicación social, n. 73, pp. 845-869.

https://doi.org/10.4185/RLCS-2018-1285

Raithel, Sascha; Schwaiger, Manfred (2015). "The effects of corporate reputation perceptions of the general public on shareholder value". Strategic management journal, v. 36, n. 6, pp. 945-956.

https://doi.org/10.1002/smj.2248

Ramírez-de-la-Piscina, Txema; González-Gorosarri, María; Aiestaran, Alazne; Zabalondo, Beatriz; Agirre, Antxoka (2014). "Periodismo de calidad en tiempos de crisis: un análisis de la evolución de la prensa europea de referencia (2001-2012)". Revista latina de comunicación social, n. 69, pp. 248-274.

https://doi.org/10.4185/RLCS-2014-1011

Roberts, Peter W.; Dowling, Grahame R. (2002). "Corporate reputation and sustained superior financial performance". Strategic management journal, v. 23, n. 12, pp. 1077-1093.

https://doi.org/10.1002/smj.274

Trumbo, Craig W.; McComas, Katherine A. (2003). "The function of credibility in information processing for risk perception". Risk analysis, v. 23, n. 2, pp. 343-353.

https://doi.org/10.1111/1539-6924.00313

Villafañe, Justo (2004). La buena reputación. Claves del valor intangible de las empresas. Madrid: Pirámide.

Villafañe, Justo (2013). La buena empresa. Propuesta para una teoría de la reputación corporativa. Madrid: Pearson Educación.

Villafañe, Justo; Bustamante, Enrique; Prado Emili (1987). Fabricar noticias. Las rutinas productivas en radio y televisión. Barcelona: Editorial Mitre. ISBN: 8476520271

Walker, Kent (2010). "A systematic review of the corporate reputation literature: definition, measurement and theory". Corporate reputation review, v. 12, n. 4, pp. 357-387.

https://doi.org/10.1057/crr.2009.26

Wartick, Stephen L. (2002). “Measuring corporate reputation definition and data”. Business and society, n. 41, pp. $371-392$. https://doi.org/10.1177/0007650302238774

Weick, Karl E.; Sutcliffe, Kathleen M.; Obstfeld, David (2008). "Organizing and the process of sensemaking". Organization science, v. 16, n. 4, pp. 409-421.

https://doi.org/10.1287/orsc.1050.0133 